\title{
The PDZ-Domain Protein Whirlin Facilitates Mechanosensory Signaling in Mammalian Proprioceptors
}

\author{
Joriene C. de Nooij, ${ }^{1}$ Christian M. Simon, ${ }^{2,4}$ Anna Simon, ${ }^{7}$ Staceyann Doobar, ${ }^{1}$ Karen P. Steel, ${ }^{8}$ ำ Robert W. Banks, ${ }^{9}$ \\ George Z. Mentis, ${ }^{2,3,4}$ Guy S. Bewick, ${ }^{7}$ and Thomas M. Jessell ${ }^{1,5,6}$ \\ Departments of ${ }^{1}$ Neuroscience, and Biochemistry and Molecular Biophysics, ${ }^{2}$ Pathology and Cell Biology, ${ }^{3}$ Neurology, ${ }^{4}$ Motor Neuron Center for Biology \\ and Disease, ${ }^{5}$ Kavli Institute for Brain Science, ${ }^{6}$ Howard Hughes Medical Institute, Columbia University, New York, New York $10032,{ }^{7}$ School of Medical \\ Sciences, University of Aberdeen, Aberdeen AB25 2ZD, United Kingdom, ${ }^{8}$ Wellcome Trust Sanger Institute, Hinxton, Cambridgeshire CB10 1SA, United \\ Kingdom, and ${ }^{9}$ School of Biological and Biomedical Sciences, University of Durham, Durham DH1 3LE, United Kingdom
}

Mechanoreception is an essential feature of many sensory modalities. Nevertheless, the mechanisms that govern the conversion of a mechanical force to distinct patterns of action potentials remain poorly understood. Proprioceptive mechanoreceptors reside in skeletal muscle and inform the nervous system of the position of body and limbs in space. We show here that Whirlin/Deafness autosomal recessive 31 (DFNB31), a PDZ-scaffold protein involved in vestibular and auditory hair cell transduction, is also expressed by proprioceptive sensory neurons ( $\mathrm{pSNs}$ ) in dorsal root ganglia in mice. Whirlin localizes to the peripheral sensory endings of pSNs and facilitates pSN afferent firing in response to muscle stretch. The requirement of Whirlin in both proprioceptors and hair cells suggests that accessory mechanosensory signaling molecules define common features of mechanoreceptive processing across sensory systems.

Key words: mechanoreception; muscle spindle; PDZ proteins; proprioceptors; sensory-motor control

\section{Introduction}

The sense of proprioception serves an essential role in sensorymotor control. Feedback from muscle is mediated by proprioceptive sensory neurons (pSNs) in the dorsal root ganglia (DRG) and originates from two main classes of mechanoreceptors: muscle spindles (MSs) and Golgi tendon organs (GTOs), innervated by Group Ia/II and Group Ib pSNs, respectively (Matthews, 1972; Pierrot-Deseilligny and Burke, 2005). The activation of these mechanoreceptors triggers afferent feedback that informs the

\section{Received Sept. 4, 2014; revised Dec. 12, 2014; accepted Jan. 6, 2015}

Author contributions: J.C.d.N., G.Z.M., G.S.B., and T.M.J. designed research;J.C.d.N., C.M.S., A.S., S.D., G.Z.M., and G.S.B. performed research; K.P.S. contributed unpublished reagents/analytic tools; J.C.d.N., R.W.B., G.Z.M., G.S.B., and T.M.J. analyzed data; J.C.d.N. and T.M.J. wrote the paper.

This work was supported by Wellcome Trust Grant 098051 to K.P.S., Medical Research Council Grant G0601253 to A.S., R.W.B., and G.S.B., Roche Young Investigator Award to C.M.S., and National Institutes of Health Grant R01NS078375 to G.Z.M. T.M.J is an Investigator of the Howard Hughes Medical Institution. We thank Ira Schieren, Barbara Han, and Susan Morton for technical help with FACS analysis and antibody generation; Andrew Miri for help with MATLAB; Monica Mendelsohn, Nataliya Zabello, and Rosalind Lacey for help with generating transgenic mice and animal breeding; H. Kremer and E. van Wijk (Radboud University Nijmegen, The Netherlands) for providing the guinea pig anti-Whrn antibody and Whrn expression construct; and Andrew Miri, Eiman Azim, and Ellen Lumpkin for comments on the manuscript.

The authors declare no competing financial interests.

This article is freely available online through the J Neurosci Author Open Choice option.

Correspondence should be addressed to any of the following: Dr. Joriene C. de Nooij, Columbia University, 701 West 168th Street, New York, NY 10032, E-mail: sd382@columbia.edu; Dr. Thomas M. Jessell, Columbia University, 701 West 168th Street, New York, NY 10032, E-mail: tmj1@columbia.edu; or Dr. Guy S. Bewick, School of Medical Sciences, University of Aberdeen, Aberdeen AB24 3FX, United Kingdom, E-mail: g.s.bewick@abdn.ac.uk

A. Simon's present address: York University, York, United Kingdom.

K.P. Steel's present address: Wolfson Centre for Age-Related Diseases, King's College London, United Kingdom. DOI:10.1523/JNEUROSCI.3699-14.2015

Copyright @ 2015 de Nooij et al.

This is an Open Access article distributed under the terms of the Creative Commons Attribution License (http://creativecommons.org/licenses/by/3.0), which permits unrestricted use, distribution and reproduction in any medium provided that the original work is properly attributed. nervous system of changes in the state of the musculoskeletal system (Pierrot-Deseilligny and Burke, 2005; Windhorst, 2007). Like many mechanoreceptor subclasses (Abraira and Ginty, 2013), the steps that underlie the conversion of mechanical deformations of pSN endings into sensory signals remain poorly understood.

Physiological studies have emphasized two prominent features of proprioceptor mechanosensation: their initial activation as well as their adaptation kinetics. The receptor potential is carried primarily by $\mathrm{Na}^{+}$, with a small contribution from $\mathrm{Ca}^{2+}$ ions (Ottoson, 1964; Hunt et al., 1978). This initial discharge activity is a function of the velocity of muscle stretch/contraction. pSNs are slowly adaptive and typically signal for the entire duration of a given stimulus (Matthews, 1972; Hunt and Ottoson, 1975; Fukami and Wilkinson, 1977). pSN stimulus-threshold sensitivity also appears regulated by autocrine glutamate signaling, which augments MS afferent sensitivity to stretch (Bewick et al., 2005). Despite these advances, the molecules that control proprioceptor excitability and firing remain unknown.

Proprioceptors express several mechanically gated ion channels, including Piezo 2 and members of the degenerin/epithelial $\mathrm{Na}^{+}$channel (DEG/ENaC) family (Simon et al., 2010; J.C.d.N. and T.M.J., unpublished data), but the role of these channels in proprioceptor sensory transduction has not been established. Moreover, these candidate transduction channels are expressed by many mechanoreceptors, as well as by nonsensory receptor cell types (Coste et al., 2010; Del Valle et al., 2012; Chen and Wong, 2013; Woo et al., 2014), and thus they are unlikely to be the sole determinants of pSN firing activity. In many instances, mechanosensory signaling also requires accessory molecules, which regulate the gating properties or membrane 
localization of the transduction channels (Xiong et al., 2012; Poole et al., 2014). Yet, the relevance of these accessory mechanotransduction molecules across sensory systems remains largely unexplored.

To investigate mechanoreceptive signaling in proprioceptors, we performed a molecular screen to identify genes expressed in MS and GTO proprioceptor subclasses. This DRG sensory neuron screen identified Whirlin/Deafness autosomal recessive 31 (DFNB31) as a proprioceptor-selective gene. Whirlin encodes a PDZ-scaffold protein implicated in sensory transduction in vestibular and auditory hair cells (Gillespie and Müller, 2009). Whirlin protein localizes to $\mathrm{pSN}$ mechanoreceptive sensory endings, and whirlin mutant mice exhibit a reduction in stretch-evoked firing frequency as well as a decreased fidelity in response to repeated stretch. These findings reveal that Whirlin facilitates pSN stretch-evoked activity, ensuring high sensitivity to muscle stretch. They also support a view that proprioceptors and hair cells rely on similar accessory scaffold molecules for aspects of mechanosensory processing.

\section{Materials and Methods}

Animal husbandry. Animal experiments were conducted according to the Institutional Animal Care and Use Committee of Columbia University, the Wellcome Trust Sanger Institute's Ethical Review Committee, and the University of Aberdeen Code of Practice of the Animal Welfare and Ethical Review Body, in accordance with the UK Animals (Scientific Procedures) Act, 1986 Amendment Regulations, 2012 (ASPA) under the authority of UK Home Office licenses. Mouse strains used for experiments were Whirler (Holme et al., 2002), Pv:Cre and Tau:loxp-STOPloxp:mGFP-nLZ (Hippenmeyer et al., 2005), and Thy1:loxp-STOP-loxp: YFP (Buffelli et al., 2003). The hEGR3:WGA-mCherry transgene was generated by a fusion of the WGA and mCherry coding sequences, which was cloned into the $h E G R 3$ promoter construct (de Nooij et al., 2013). Transgenic mice were generated by pronuclear injection. Mice heterozygous or homozygous mutant for the $\mathrm{Whrn}^{w i}$ allele, or carrying reporter transgenes, were identified by DNA genotyping (details available upon request).

Isolation of DRG neuronal subsets through FACS. DRGs were dissected in ice-cold Hank's balanced salt solution (HBSS) and dissociated by enzymatic digestion (Papain, Collagenase 2, Dispase type II; Worthington Biochemical), followed by trituration, essentially as described previously (Malin et al., 2007). Cell suspensions were passed through $35 \mu \mathrm{m}$ gauze filters to clear suspension from remaining cellular aggregates. Fluorescently labeled neuronal subsets were isolated through FACS using a FACSAria II flow cytometer (BD Biosciences; Irving Cancer Center Flow Cytometry Core) or Beckman Coulter Altra Hypersort (BD Biosciences). Neurons were sorted at $12-13$ psi using a $100 \mu \mathrm{m}$ nozzle and collected in $\mathrm{HBSS} / 1 \%$ FBS. Neuronal samples were pelleted and stored at $-80^{\circ} \mathrm{C}$ until processed.

Affymetrix gene chip analysis. MS and GTO pSNs were obtained from dissociated DRGs of p7-p10 hEGR3:WGA-mCherry; Pv-Cre; Thy1:loxpSTOP-loxp:YFP mice of either sex using FACS (see Fig. 1). To obtain at least three cRNA samples ( $\sim 3-5 \mu \mathrm{g}$ each) for both MS and GTO neuronal subsets, neurons were pooled from multiple FACS experiments. RNA was extracted from MS and GTO neuronal subsets (Absolutely RNA nanoprep kit; Agilent Technologies) and prepared for Gene Chip analysis using Ovation Pico RNA amplification, and cDNA Biotin Module V2 kits (NuGen). Hybridization of fragmented biotin-labeled cRNA was performed by the Columbia Genome Core Facility using GeneChip Mouse Genome 430 2.0 Arrays (Affymetrix). Raw data from CEL files (deposited in NCBI's GEO database (Edgar et al., 2002); accession GSE64941) were analyzed with Partek Genomic Suite 6.6 software (Partek), using GCRMA background correction. Principle component analysis of all samples indicated good correlation between MS neuronal samples but very poor correlation between GTO neuronal samples, for reasons unknown. Because of the high variability between GTO samples, we arbitrarily set the stringency levels of the statistical threshold for all our analyses to $p<0.08$. In addition, we limited the analysis to genes that were enriched in the MS neuronal subset compared with the GTO neuronal subset, and used a cutoff of fivefold or higher. Using these parameters, our analysis resulted in the identification of 626 differentially expressed Affymetrix probe sets. All differentially expressed probe sets were validated, to a first degree, using the Allen Spinal Cord Atlas (Allen Institute for Brain Science) to assess their abundance of expression in DRG. Transcripts that were expressed in relatively small subsets of DRG neurons in the Allen Spinal Cord Atlas (i.e., showed expression patterns that were either similar to the pan-pSN marker Runx3, or showed expression in fewer numbers of neurons than Runx3; 46 in total) were selected for further analysis.

A more recent understanding of the Pv-expression pattern in DRG (de Nooij et al., 2013) suggests that the (Pv:)YFP-only population includes a population of cutaneous mechanosensory sensory neurons. Some of these may innervate sensory receptors in which the $h E G R 3$ promoter may also be active. Therefore, both MS and GTO neuronal subsets are likely "contaminated" by subsets of cutaneous neurons, limiting the effectiveness of our screen. This, together with the poor chip hybridization results of the GTO neuronal samples, resulted in a relatively high proportion of pan-pSN markers (including Whrn) because transcripts from GTO-innervating neurons were represented at much reduced levels.

In situ hybridization, immunohistochemistry, and semiquantitative RT-PCR. In situ hybridization and immunohistochemistry were performed as described previously (de Nooij et al., 2013). Primary antibodies used in immunohistochemistry experiments were as follows: $\mathrm{Rb}$ and Gp anti-Runx3 (Chen et al., 2006; Kramer et al., 2006), Gt anti-Pv (Swant), Ck anti- $\beta$-galactosidase (Abcam), Shp anti-GFP (Biogenesis), Gp and Rb anti-vGluT1 and Gt anti-Chat (Demireva et al., 2011), Rb anti-Shankla (Betley et al., 2009), and Gp anti-Whrn (van Wijk et al., 2006). A Rb anti-Whrn antibody was generated against the same GST fusion protein as the Gp antibody, using an expression construct obtained from H. Kremer (Radboud University Nijmegen, The Netherlands). The $\mathrm{Rb}$ anti-Whrn antibody was affinity-purified using a glutathione-agarose column coupled to the GST-Whrn fusion protein and assessed for specificity using wi/wi mutant tissue. Fluorophoreconjugated secondary antibodies were obtained from Jackson ImmunoResearch Laboratories and Invitrogen. Images were acquired on a Zeiss Axioskop2 or Zeiss LSM510 Meta confocal microscope.

For semiquantitative PCR, RNA was isolated from whole DRG or purified (FACS) fluorescently labeled $\mathrm{Pv}^{+}$sensory neurons (Absolutely RNA mini/nanoprep kits; Agilent Technologies). Following first stand synthesis (Superscript III; Invitrogen), semiquantitative PCR analysis was performed using primers specific for Whrn- $L$ and Whrn-S isoforms. Primer sequences were as follows: Whrn-Lf, $5^{\prime}$-ATTCTGGAGGTGAATG GGCG-3'; Whrn-Lr, 5' -TCAAAGCGGTCCAGGTCTTG-3'; Whrn-S1f, 5'-CATCGTCCCAGGGTTAGGTG-3'; Whrn-S1r, 5' $^{\prime}$-CTGGGGGTCTC CATAGGTCA-3'; Whrn-S2f, 5'-GATGCGAGCACTTTGTACGC-3'; and Whrn-S2r, 5'-ACAGGAGTGAGAACTTGGCG-3'. Input cDNA was $2 \mathrm{ng}$, and PCRs were run for 30 cycles with an annealing temperature of $58^{\circ} \mathrm{C}$.

Neuronal and muscle sensory ending counts. Neuronal cell counts were performed on serial cryostat sections $(30 \mu \mathrm{m})$ of individual DRG, including all sections in counts. Neurons or nuclei with $<40 \%$ of normal cellular/nuclear surface area were excluded from counts to avoid double counting of neurons at the plane of sectioning. Counts of vGluT1 ${ }^{+}$sensory endings were performed on whole-mount soleus muscles.

Conduction velocity and sensory-motor connectivity assays. Conduction velocity, $\mathrm{H}$-reflex, and ventral root compound action potentials were recorded from p12-p13 (the latest time points at which these recordings are feasible in vitro) wild-type, and wi/wi mutant litter mates of either sex, as described previously (Shneider et al., 2009; Mentis et al., 2011). In brief, animals were anesthetized by hypothermia, decapitated, and the spinal cords were dissected and removed under cold $\left(9^{\circ} \mathrm{C}\right) \mathrm{aCSF}$ containing the following (in mM): $128.35 \mathrm{NaCl}, 4 \mathrm{KCl}, 0.58 \mathrm{NaH}_{2} \mathrm{PO}_{4}-\mathrm{H}_{2} \mathrm{O}, 21$ $\mathrm{NaHCO}_{3}, 30$ D-glucose, $1.5 \mathrm{CaCl}_{2}-\mathrm{H}_{2} \mathrm{O}$, and $1 \mathrm{MgSO}_{4}-7 \mathrm{H}_{2} \mathrm{O}$. The sciatic nerve was dissected in continuity with its parent ventral and dorsal roots (L4-L5) to the hindlimb. The spinal cord-hindlimb preparation was then transferred to a customized recording chamber and perfused continu- 
ously with oxygenated $\left(95 \% \mathrm{O}_{2} / 5 \% \mathrm{CO}_{2}\right)$ aCSF (flow rate: $\sim 10 \mathrm{ml} / \mathrm{min}$, at $\sim 22^{\circ} \mathrm{C}$ ). The $\mathrm{L} 3$ dorsal and ventral roots were placed into suction electrodes for stimulation and recording, respectively, of the sensorymotor spinal reflex (see Fig. 5). A bipolar "hook" electrode was placed on the sciatic nerve for "en passant" stimulation. A bipolar concentric needle was inserted into the tibialis anterior (TA) muscle to record EMG activity. Finally, a suction electrode was placed on the L4 DRG to record extracellularly the incoming volley from activated sensory neurons following sciatic nerve stimulation. The conduction velocity of the fastest sensory afferents (i.e., proprioceptive) was calculated from the distance between the TA EMG electrode to the electrode placed in the L4-DRG divided by the latency of the first spike (peak) recorded from the L4-DRG suction electrode following stimulation of the TA muscle. The maximum amplitude of the dorsal-to-ventral root compound action potential was achieved by dorsal root stimulation at $\sim 5 \times$ threshold. The H-reflex recorded in the TA muscle was elicited at different stimulation intensities in the sciatic nerve. H-reflex latency was measured from the onset of the stimulation artifact to the onset of the H-reflex.

Soleus muscle stretch assays. Muscle afferent stretch assays were performed essentially as described previously (Bewick et al., 2005). Wildtype and wi/wi mutant adult animals of either sex were killed by cervical dislocation, and soleus ( $\mathrm{Sol}$ ) muscles and associated tendons and muscle nerve were dissected free in oxygenated Liley's containing the following (in mM): $138.8 \mathrm{NaCl}, 4 \mathrm{KCl}, 12 \mathrm{NaHCO}_{3}, 1 \mathrm{KH}_{2} \mathrm{PO}_{4}, 1 \mathrm{MgCl}_{2}, 2 \mathrm{CaCl}_{2}$, and 11 glucose (Liley, 1956). Sol muscles were chosen for experiments because of their size and long distal tendon, which renders them well suited for ex vivo recordings. Muscles were mounted with the fibular insertion pinned securely to the bottom of a Sylgard (Dow Corning)-coated dish and the calcaneal tendon attached to a micromanipulator with a vernier scale (Prior Scientific). Whole-muscle nerve recordings were performed extracellularly, using glass fire-polished suction electrodes. Signals were amplified (Neurolog NL104 preamplifier) and bandpass filtered (200-2000 Hz, Neurolog NL125, both Digitimer) and recorded through a PCI-6221 interface (National Instruments) using WinWCP software (Strathclyde Electrophysiological Software, Strathclyde University).

Before recordings, resting/baseline muscle length (to measure spontaneous activity) was set with extreme care. Resting length was defined as the muscle length at which firing did not change when the muscle was allowed to shorten from that length, whereas, conversely, firing increased following the smallest detectable increase in muscle length (stretch). Typically, multiple "set lengths" were tested until reproducibility was achieved. Once set, resting length approximated in vivo resting length at $90^{\circ}$ ankle flexion measured during dissection and was generally constant throughout the duration of the experiment. In situ muscle length for wildtype and wi/wi mutant Sol muscles at full ankle extension and at midextension $\left(90^{\circ}\right.$ ankle) was nearly identical at $\sim 10 \mathrm{~mm}$ (data not shown). A 1 $\mathrm{mm}$ increase in muscle length thus corresponds to a similar length increase for both wild-type and wi/wi mutant Sol muscles. Stretch-evoked and spontaneous afferent firing frequencies were recorded during 3 trials; each trial consisting of 4 repeats of a $5 \mathrm{~s}$ muscle stretch of $1 \mathrm{~mm}$ length, followed by a $5 \mathrm{~s}$ period during which muscles were returned to resting length (see Fig. $4 A$ ). Individual trials were spaced $\sim 15 \mathrm{~min}$ apart; and in between trials, muscles were unloaded. Muscles were totally unloaded for $30 \mathrm{~min}$ before experiments and during drug incubations (30-40 min).

Recordings were analyzed using Clampfit10.3 data analysis software (Molecular Devices). Per trial, data for evoked-stretch responses were obtained from stretch episodes s2-s4 (see Fig. 4); data from the first stretch were omitted to exclude putative effects from the extended rest during the intertrial interval ( $\mathrm{s} 1$ data were used for variability analysis; see Fig. 7). Data for spontaneous activity were obtained from the episodes preceding stretch episodes 2,3 , and 4 . Spike counts were performed on $1 \mathrm{~s}$ intervals centered within stretch episodes to restrict spike counts to the static phase of the muscle stretch. Spike thresholds were set manually, generally within the $1-2 \times$ SD threshold range. The net-evoked activity of stretch episodes was calculated by subtracting the spontaneous activity spike count (of the preceding episode at resting length) from the activity spike count recorded during stretch. Net-evoked activity was averaged per trial, and the overall mean net evoked activity per muscle was averaged from the number of trials included in the analysis (generally 3 ).
Among stretch-responsive muscles, we distinguished between two categories: those Sol muscles that exhibited clear spontaneous activity when the muscle was held at baseline length (Category 1 ) and those that had no or negligible levels ( $<10 \mathrm{imp} / \mathrm{s}$ ) of spontaneous activity (Category 2$)$. We find that the percentages of Category $1(\mathrm{C} 1)$ and Category $2(\mathrm{C} 2)$ muscle are similar for wild-type and wi/wi mutants ( $\mathrm{C} 1$ in wt $39.1 \%$ and $34.6 \%$ in wi/wi). By comparing the net stretch-evoked activity levels of $\mathrm{C} 1$ and C2 muscles for both wild-type and wi/wi Sol afferents, we find that a lack of spontaneous activity does not correlate with a reduced level of netevoked activity (wt $\mathrm{C} 1$ vs $\mathrm{C} 2 p=0.312, t$ test; wi/wi $\mathrm{C} 1$ vs $\mathrm{C} 2 ; p=0.977$, $t$ test) (data not shown). This justifies the inclusion of both $\mathrm{C} 1$ and $\mathrm{C} 2 \mathrm{Sol}$ muscles in our analysis described in Figures 4, 6, and 7. In addition, we found that for wild-type Sol pSNs, stretch-evoked discharge activity did not correlate with age, sex, or weight of the animals (data not shown).

Statistical analysis. All statistical analysis was performed using SigmaPlot 11.0 (Systat Software) with significance levels set at $\alpha \leq 0.05$. For calculation of the coefficient of variation (CV), spike counts per $100 \mathrm{~ms}$ bins of each stretch episode (s2-s4 of all trials) were determined using MATLAB (MathWorks). The CV of an individual stretch episode was calculated by dividing the SD by the mean spike frequency of all $100 \mathrm{~ms}$ bins. The CV per Sol muscle was calculated as the mean CV of all stretch episodes.

\section{Results}

\section{Identification of Whirlin as a pSN-specific molecule in DRG} sensory neurons

To identify genes involved in sensory transduction in pSNs, we isolated transcripts expressed in proprioceptor subtypes. Our screen relied on a Egr3:WGA-mCherry transgenic line in which a human EGR3 promoter element directs expression of a wheat germ agglutin-mCherry fusion protein (WGA-mCherry) to intrafusal muscle fibers and, through uptake by their sensory endings, labels Group Ia/II muscle spindle afferent cell bodies in DRG (Fig. 1A) (de Nooij et al., 2013). In the presence of an additional fluorescent reporter (YFP or GFP) driven by the Parvalbumin locus (thus labeling all pSNs), WGA-mCherry uptake by MS afferent terminals provides a way of distinguishing MS- and GTO-innervating pSNs through FACS (Fig. $1 A, B$ ). Isolated pSN populations were processed for Affymetrix-based microarray analysis. This approach yielded several candidate molecules that appeared enriched in subsets, or in all pSNs, as assessed using the Allen Spinal Cord Atlas (Allen Institute for Brain Science) (Fig. $1 C)$. Many of these genes encode signaling molecules, only a few of which (Sema3d, Cdh13) have previously been described in association with a pSN identity (Fig. 1C) (Takahashi et al., 2009; Poliak et al., in preparation). Here we report on one of the panpSN transcripts, Whirlin (Whrn)/Deafness autosomal recessive 31 (DFNB31). Whrn encodes a PDZ-scaffold protein required for the function of retinal photoreceptors, as well as vestibular and cochlear hair cells (Mburu et al., 2003; Yang et al., 2010) and which, by virtue of its localization at stereocilia tip links, has been implicated in mechanotransduction (Gillespie and Müller, 2009).

The requirement of Whrn in hair cells and photoreceptors raised the question of its role in sensory signaling in proprioceptors. We analyzed the developmental pattern of Whrn expression in pSNs, as well as its subcellular localization. Expression of Whrn transcript in DRG was first observed at embryonic day (e) 15.5 (data not shown), and by postnatal day (p) 0 , we found that $77 \pm$ $2 \%$ of $\mathrm{Whrn}^{+}$neurons coexpressed the runt-transcription factor Runx3 (Rx3) and Parvalbumin (Pv), markers that define proprioceptive neuronal identity (Fig. 2A,C) (de Nooij et al., 2013). Conversely, $94 \pm 2 \%$ of pSNs expressed Whrn protein (Fig. $2 E$ ), indicating that Whrn expression at early developmental stages is largely confined to proprioceptive sensory neurons. During the first postnatal week, the level of Whrn expression in sensory neurons increased and remained at high levels throughout adult life 
A

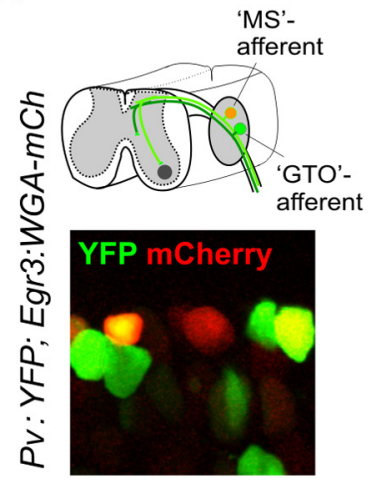

D

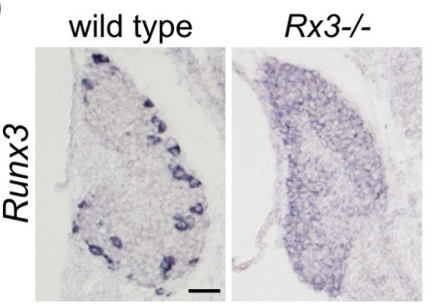

B

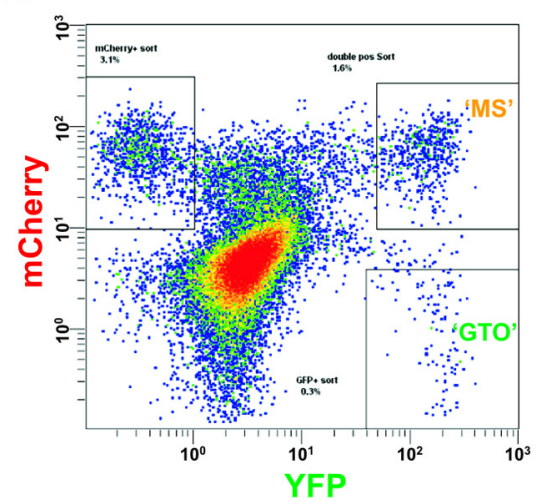

C

\begin{tabular}{|l|l|r|}
\hline symbol & gene name & fold change \\
\hline Creg2 & cellular repressor of E1A-stimulated genes & 48.3 \\
\hline Otud7b & deubiquitinase & 25.3 \\
\hline Pcdhb9 & protocadherin beta 9 & 21 \\
\hline Nrip1 & nuclear receptor interacting protein 1 & 17.4 \\
\hline Pogz & pogo transposable element with ZNF domain & 16.4 \\
\hline Arhgef9 & Cdc42 guanine nucleotide exchange factor (GEF) 9 & 14.6 \\
Pvrl3 & poliovirus receptor-related 3 (nectin3) & 13.7 \\
\hline Cdh13 & Cadherin 13 & 13.5 \\
Lrrc4c & leucine rich repeat containing 4C (NG1-L) & 11.1 \\
\hline Trhde & thyrotropin-releasing hormone degrading enzyme & 9.7 \\
Sema3d & Semaphorin 3d & 8.5 \\
Wnt7a & wingless-type MMTV integration site fam., member 7A & 7.4 \\
Cgnl1 & cingulin-like 1 & 7 \\
Sulf1 & sulfatase 1 & 6.8 \\
Slc35c1 & solute carrier family 35, member C1 & 6.7 \\
Lppr5 & lipid phosphate phosphatase-related protein type 5 & 6.3 \\
Whrn & Whirlin & 6.2 \\
Onecut2 & one cut homeobox 2 & 6.2 \\
Asb13 & ankyrin repeat and SOCS box containing 13 & 6.1 \\
\hline Rasgrf2 & RAS prot.-specific guanine nucleotide-releasing factor 2 & 6 \\
Sorcs2 & sortilin-related VPS10 domain containing receptor 2 & 5.8 \\
Reg3b & regenerating islet-derived 3 beta & 5.7 \\
Plekhg1 & pleckstrin homology dom. containing, fam. G, member 1 & 5.6 \\
Cnr1 & cannabinoid receptor 1 & 5.6 \\
Tspan2 & tetraspanin 2 & 5.4 \\
Cntn3 & contactin 3 & 5.2 \\
Grm8 & glutamate receptor, metabotropic 8 & 5.1 \\
\hline
\end{tabular}

Figure 1. Identification of transcripts enriched in MS and GTO-innervating proprioceptors. A, Genetic strategy to fluorescently label MS- and GTO-innervating pSN subtypes based on Pv:Cre, Thy1:Ioxp-stop-loxp:YFP, and hEGR3:WGA-mCherry transgenic mice. Pv:Cre-induced expression of the YFP-reporter (Pv:YFP) labels all proprioceptors with YFP; expression of $h E G R 3$ :WGA-mCherry in intrafusal muscle fibers selectively labels MS-innervating afferents by virtue of their accumulation of WGA-mCherry. The $h E G R 3$ promoter also activates expression of WGA-mCherry in the end organs of some classes of cutaneous sensory neurons (resulting in YFP ${ }^{-}$mCherry ${ }^{+}$neurons). B, FACS profile of dissociated DRG neurons obtained from Pv:YFP; hEGR3:WGA-mCherry mice. YFP ${ }^{+}$, "MS," and "GTO" pSN subsets are segregated based on the differential labeling with mCherry. $x$ - and $y$-axes represent YFP and mCherry fluorescence intensity, respectively. $C$, A selection of the 46 identified transcripts enriched in "MS"-innervating pSNs, which show expression patterns in DRG that are similar to the pattern of the pan-pSN marker Runx3 or that are expressed in smaller neuronal subsets. The selected transcripts listed exhibited the more abundant expression levels when assessed through the Allen Spinal Cord Atlas (Allen Institute for Brain Science) or through in situ hybridization analysis. D, Expression of Runx 3 and Whrn in p0 DRG. Whrn, one of the candidate PSN markers identified, resembles the Runx3 expression pattern in wild-type DRG (indicative of expression in all or subsets of pSNs) but is absent in Runx $3^{-/-}$mutant DRG.

(Figs. $2 B$ and $3 A$ ). Although Whrn was originally selected on the basis of its enriched expression in MS-innervating pSNs, at these later developmental stages ( $\geq \mathrm{p} 8$ ), Whrn was expressed in $95 \pm$ $1 \%$ of pSNs, indicating that it is expressed in both Group Ia/II MS afferents and Group Ib GTO afferents (Fig. 2E). More strikingly, Whrn protein appeared to be confined to pSNs (Fig. 2C,D). Thus, in contrast to other documented markers for pSNs, $\mathrm{Rx} 3, \mathrm{Pv}$, TrkC, and Etv1, which are shared with subsets of cutaneous sensory neurons (de Nooij et al., 2013), Whrn appears selectively expressed in proprioceptors in postnatal DRG.

Whrn is required for multiple cellular functions in hair cells and photoreceptors, and mutations in the Whrn gene are associated with visual, auditory, and vestibular impairment (Mburu et al., 2003; Maerker et al., 2008; Yang et al., 2010). The severity of sensory impairment varies with the mutation and the level of expression of the two main Whrn isoforms. Vestibular and cochlear hair cells express both the long isoform (Whrn-L), which contains two N-terminal PDZ domains, a proline-rich domain (PRD) and a C-terminal PDZ domain, and the short C-terminal isoform (Whrn-S), containing just the PRD and C-terminal PDZ domain (Fig. 2F) (Mburu et al., 2003). Photoreceptors, in contrast, express only Whrn-L (Yang et al., 2010). Mutations that affect the $\mathrm{N}$-terminal PDZ domains primarily result in the degeneration of photoreceptors (Yang et al., 2010). Mutations that affect the C-terminal PRD and PDZ domains appear dispensable for photoreceptor function but instead cause morphological abnormalities, and eventual degeneration, of both cochlear and vestibular hair cells (Holme et al., 2002; Yang et al., 2010).
To determine which Whrn isoforms are expressed by pSNs, we performed a semiquantitative RT-PCR analysis using primer sequences specific for either the Whrn-L or Whrn-S isoform. Whrn-L, but not Whrn-S, was detected in whole DRG or purified $\mathrm{Pv}^{+}$DRG sensory neurons (Fig. $2 F$ ). Thus, in contrast to hair cells, but as with photoreceptors, pSNs appear to express exclusively the long Whrn isoform.

We also examined the subcellular distribution of Whrn in pSNs. We detected Whrn protein in MS and GTO peripheral sensory endings (Fig. 2G; and data not shown) but did not detect expression of Whrn transcript in MS intrafusal muscle fibers or associated support cells (data not shown). This suggests that, in the MS, Whrn expression is concentrated in pSN terminals. Within the spinal cord, we failed to detect Whrn protein in proprioceptor vGluT1 $^{+}$synaptic terminals on spinal motor neurons (Fig. $2 H$ ), although low levels of Whrn were detected in spinal neurons. The apparent absence of Whrn in pSN central terminals may reflect a difference in the subcellular stabilization and/or translocation of Whrn protein between peripheral and central pSN axonal branches. The restricted expression of Whrn in pSNs and its localization to the peripheral sensory terminals prompted us to analyze the functional role of Whrn in proprioceptor mechanosensation.

\section{Whirlin is not required for the development or maintenance of proprioceptors}

To determine whether Whrn is required for the development or maintenance of proprioceptors, we assessed pSN morphology in homozygous whirler ( $W h r n^{w i}$, abbreviated to wi) mutant mice. wi/wi mutants harbor a deletion of amino acids $434-631$ of the 
A

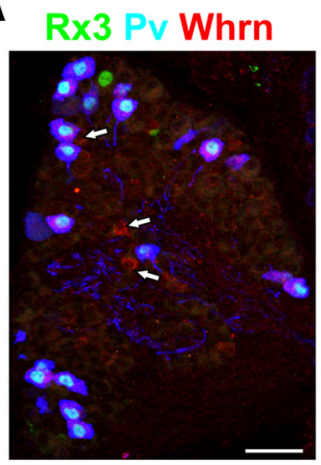

C

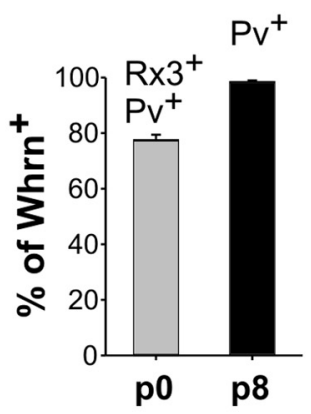

Whrn

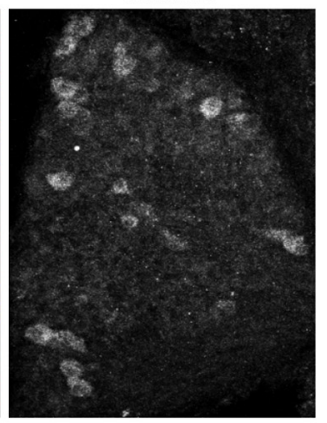

D

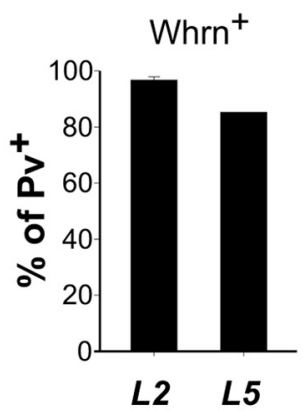

B
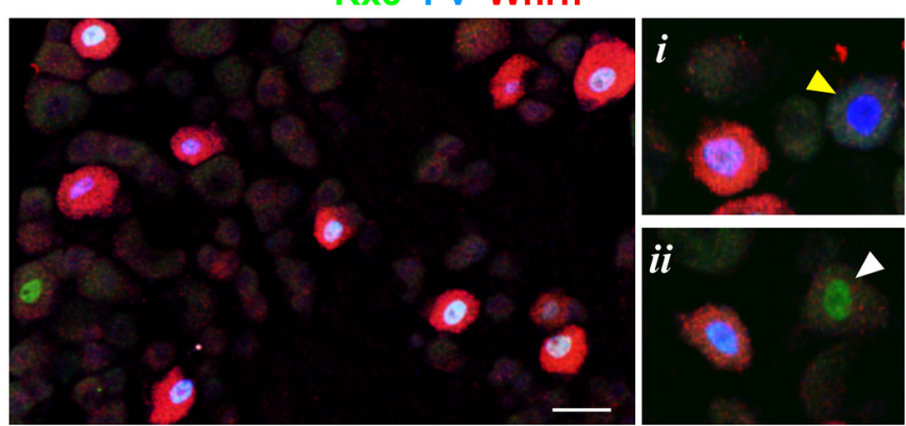

E

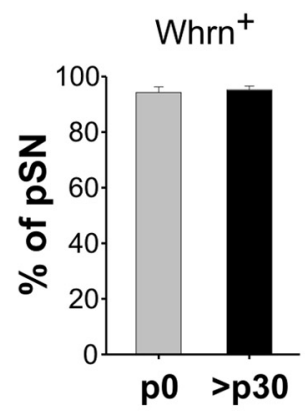

F

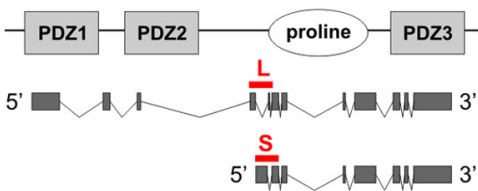

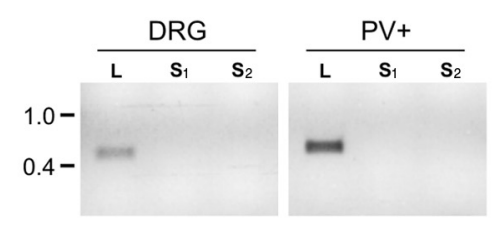

G

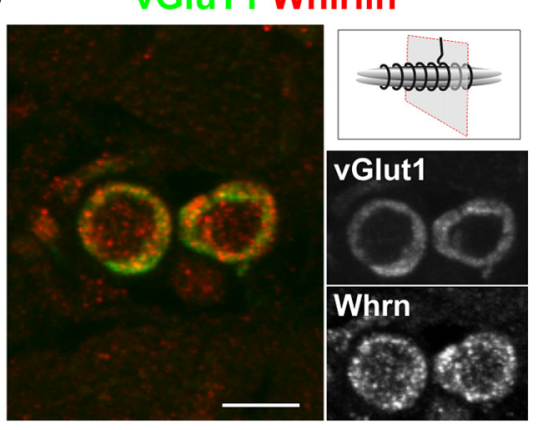

H

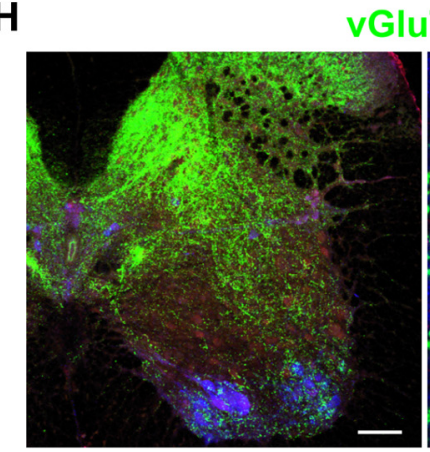

vGluT1 Chat Whirlin

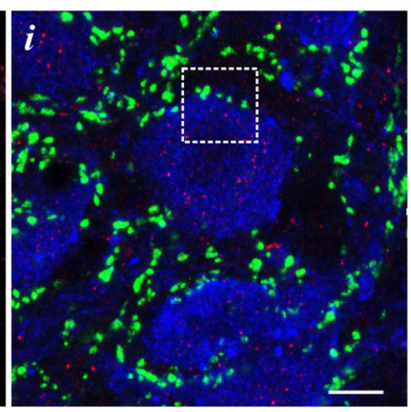

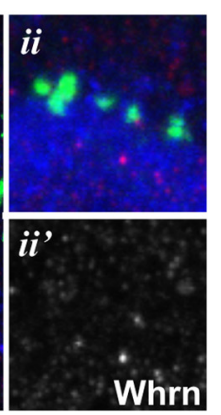

Figure 2. Selective expression of Whrn in proprioceptive sensory neurons. $A$, Expression of Whrn, Rx3, and Pv protein in DRG at p0. Although most Whrn ${ }^{+}$neurons coexpress Rx3 and Pv, at this stage a few Whrn ${ }^{+}$neurons do not (arrows). B, Expression of Whrn, Rx3, and Pv in p8 DRG. At this stage, Whrn is only expressed in sensory neurons that coexpress Rx3 and Pv, indicating that its expression in DRG is confined to pSNs and is absent from cutaneous mechanoreceptive sensory neurons that express Pv or Rx3 individually (i,iii, yellow and white arrowheads). Pv expression was assessed using the nuclear laCZ (nLZ) reporter in Pv:Cre; Tau:loxp-STOP-loxp:mGFP-nLZ mice. C, Percentage of Whrn ${ }^{+}$neurons that coexpress Rx3 and Pv (p0; $77.4 \pm 2.0 \%, n=81$ Whrn ${ }^{+}$neurons) or Pv (p8; $98.4 \pm 0.6 \%, n=415 \mathrm{Whrn}^{+}$neurons). $D$, Percentage of Pv neurons expressing Whrn in L2 and L5 DRG at p8. At this stage, the percentages of Whrn ${ }^{+}$PV neurons in L2 and L5 DRGs are similar to the percentages of Rx3 ${ }^{+}$PV neurons (de Nooijetal., 2013), supporting theidea that expression of Whrn is confined to $\left(\mathrm{Rx}^{+}{ }^{+} \mathrm{Pv}{ }^{+}\right) \mathrm{pSN}$. E, Percentage of Rx3 ${ }^{+} \mathrm{PV}{ }^{+} \mathrm{pSNs}$ that coexpress Whrn at p0 $(94.3 \pm 2.0 \%$, $n=67 \mathrm{Rx} 3{ }^{+} \mathrm{Pv}^{+}$neurons) and $>\mathrm{p} 30\left(95.2 \pm 1.4 \%, n=361 \mathrm{Rx} 3^{+} \mathrm{Pv}^{+}\right.$neurons). $\boldsymbol{F}$, Top, Schematic rendering of the Whrn protein (consisting of two N-terminal PDZ-domains, a proline-rich domain, and a (-terminal PDZ-domain) and the major Whrn isoforms. Red bars represent isoform-specific domains targeted in RT-PCR analysis. Bottom, RT-PCR analysis of the whrn long (L) and short (S) isoforms (assessed by two different primer sets, $\mathrm{s} 1$ and s2), both in whole DRG, as well as in purified $\mathrm{Pv}^{+}$sensory neurons, indicating that pSNs do not express the short C-terminal Whrn isoform. Consistent with these results, using an antibody directed against the C-terminal portion of the protein, we were unable to detect Whrn protein in DRG of a conditional Whirlin mouse mutant, which selectively perturbs the Whirlin-L isoform (data not shown). DNA marker indicates size in kilobases. G, Transverse section through a muscle spindle at p12 indicating that Whrn protein localizes to vGluT1 ${ }^{+}$pSN sensory terminals. Schematic indicates plane of section. $\boldsymbol{H}$, Expression of Whrn, vGluT1, and ChAT in p12 spinal cord. $\boldsymbol{i}$, ii (boxed area in $\boldsymbol{i}$ ), and $\mathbf{i}{ }^{\prime}$, Details show high-power images of ChAT ${ }^{+}$motor neurons studded with vGluT $1^{+}$pSN sensory terminals. Whrn protein is not observed in synaptic termini. Error bars indicate SEM. Scale bars: $\boldsymbol{A}, \boldsymbol{B}, \boldsymbol{E}, 20 \mu \mathrm{m} ; \boldsymbol{H}, 100 \mu \mathrm{m} ; \boldsymbol{H i}, 10 \mu \mathrm{m}$.

Whrn-L isoform, which introduces a premature stop codon that truncates Whrn-L after the two N-terminal PDZ-domains and disrupts expression of Whrn-S (Fig. 2F) (Mburu et al., 2003; Yang et al., 2010). In these wi/wi mutant mice, both cochlear and vestibular hair cells eventually degenerate, associated with profound vestibular-motor dysfunction (Holme et al., 2002).

We found that $\mathrm{pSNs}$ are present in normal numbers in adult wi/wi mutants (Fig. 3A,B). Moreover, in wi/wi mutants, pSNs give rise to intraspinal axonal projections and synaptic terminals that are anatomically indistinguishable from their wild-type lit- termates (Fig. 3C,D). Likewise, we observed no overt differences in the morphology or in the number of MS or GTO peripheral sensory endings in wi/wi mutants (Fig. $3 E-G$ ). The absence of overt developmental abnormalities in wi/wi mutant pSNs argues against a major role for Whrn in pSN maintenance.

Muscle spindle stretch-evoked impulse activity is reduced in wi/wi mutant mice

To assess whether Whrn functions in pSN mechanosensory signaling, we examined spontaneous and stretch-evoked discharge activity 
A
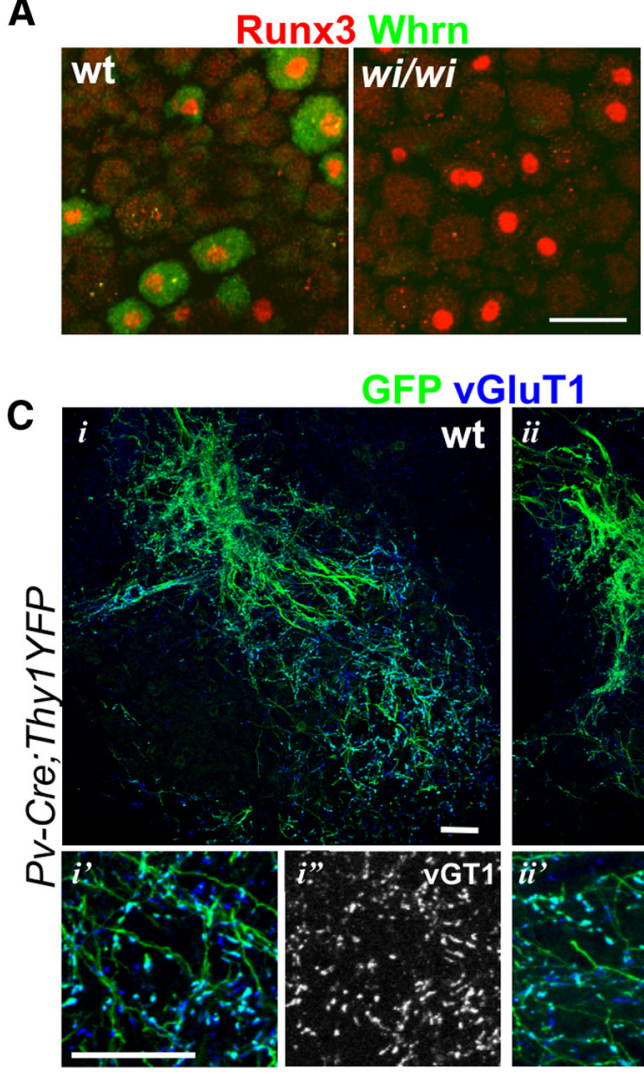

GFP vGluT1

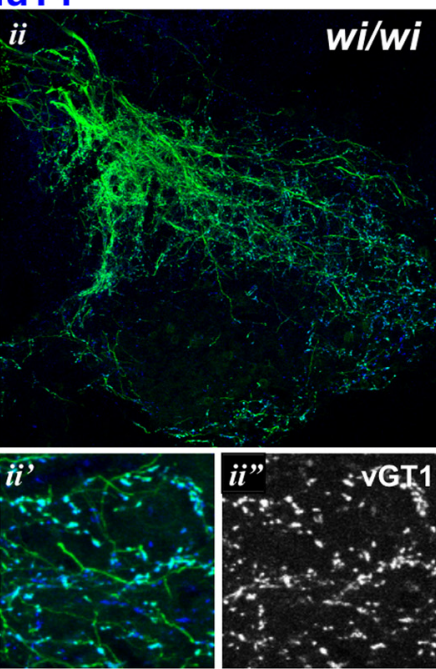

D
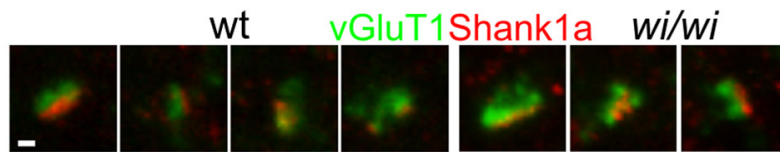

E

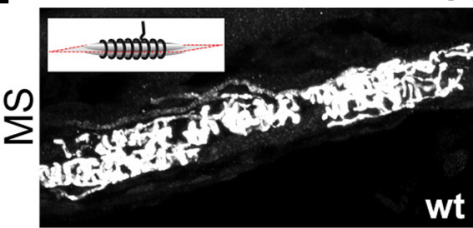

vGluT1
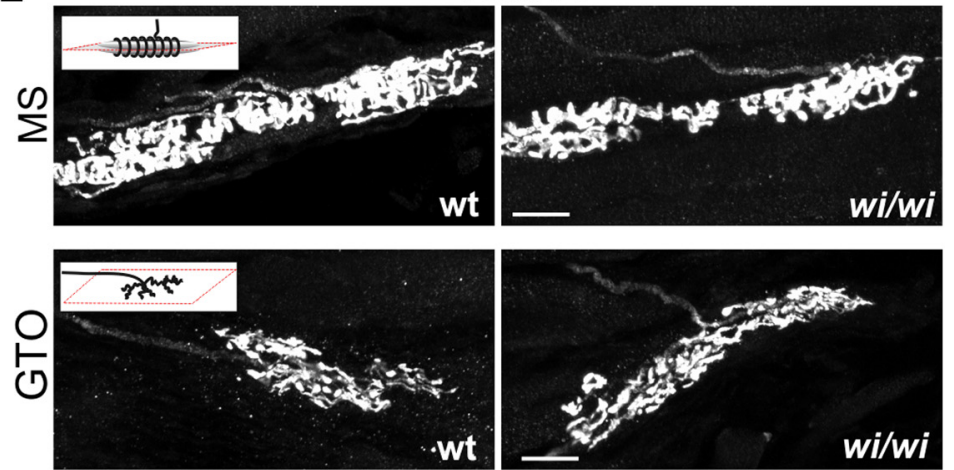

$\mathbf{F}$

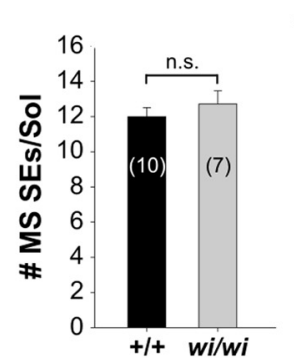

B

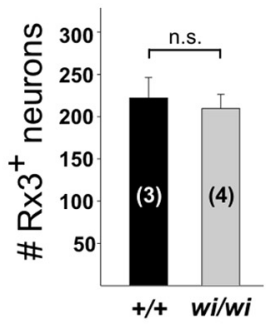

in adult wild-type and wi/wi mutant pSNs. We measured $\mathrm{pSN}$ afferent firing frequencies using extracellular recordings from soleus (Sol) muscle nerve, during both muscle stretch and at rest (Bewick et al., 2005). Individual Sol muscles were subjected to three stretch trials (t1-t3), which were spaced $\sim 15$ min apart. Each trial consisted of four consecutive stretch episodes (s1-s4; $1 \mathrm{~mm}, \sim 5 \mathrm{~s}$ each) separated by $\sim 5$ $\mathrm{s}$ intervals ( $\mathrm{r} 2-\mathrm{r} 4$, with $\mathrm{r} 1$ preceding the first stretch) during which muscles were kept at resting length (Fig. 4A) (for details, see Materials and Methods). In all trials, Sol muscle nerve firing frequencies were recorded during stretch episodes s1-s4 and during the preceding rest intervals (r1-r4). For the following analysis, we omitted the data obtained from stretch episode $s 1$ because of the extended restperiod preceding each trial.

We observed no differences in spontaneous discharge activity (SA) between wild-type and wi/wi mutant Sol pSNs $\left(\right.$ mean SA ${ }^{\text {r2-r4 }} \pm$ SEM for wt $12 \pm 3 \mathrm{im}-$ pulses per second (imp/s), for wi/wi $9 \pm 3$ $\mathrm{imp} / \mathrm{s} ; p=0.319$, Mann-Whitney rank sum test) (Fig. 4B). In contrast, stretchevoked responses recorded from Sol pSNs differed markedly between wild-type and wi/wi mutant animals. Net-evoked discharge activity (NEA; evoked activity minus the SA of the preceding interval at resting length) in wi/wi mutant Sol pSNs was reduced by $50 \%$ compared with wildtype (mean NEA ${ }^{\text {s2-s4 }} \pm$ SEM for wt $103 \pm$ $9 \mathrm{imp} / \mathrm{s}$, for $w i / w i 52 \pm 7 \mathrm{imp} / \mathrm{s} ; p<0.001$, Mann-Whitney rank sum test) (Fig. 4C). Heterozygote $+/ w i$ animals showed a reduction of $25 \%$ in NEA compared with wild-type (mean NEA ${ }^{\text {s2-s4 }} \pm$ SEM for +/wi $77 \pm 13 \mathrm{imp} / \mathrm{s} ; p=0.019$; MannWhitney rank sum test) (Fig. $4 C$ ), suggesting that the impairment in the afferent stretch response correlates with the level of Whrn protein. Thus, Whrn expression facilitates stretch-evoked discharge activity in Sol muscle nerve.

The reduction in stretch-evoked pSN firing in wi/wi mutants could result from a specific perturbation at the MS sensory ending or a general reduction in $\mathrm{pSN}$ excitabil-

wild-type and wi/wi mutant mice $\boldsymbol{E}_{\text {v }} \mathrm{GGluT1}{ }^{+} \mathrm{MS}$ and GTO sensory endings in p10 gluteus muscle in wild-type and wi/wi mutant mice. $\boldsymbol{F}$, Number of MS sensory endings (SEs) is similar in wild-type and wi/wi mutant Soleus (Sol) muscle ( $p=$ $0.428, t$ test). G, Number of GTO SEs is similar in wild-type and wi/wi mutant Sol muscles ( $p=0.471, t$ test). Number of DRG $(\boldsymbol{B})$ or muscles $(\boldsymbol{F}, \boldsymbol{G})$ analyzed is indicated in parentheses. Error bars indicate SEM. n.S., Not significant. $\boldsymbol{E}$, Schematic insets, Plane of section. Scale bars: $\boldsymbol{A}, \boldsymbol{E}, 20 \mu \mathrm{m} ; \boldsymbol{D}, 1 \mu \mathrm{m} ; \boldsymbol{C}, 50 \mu \mathrm{m}$.

Figure 3. Proprioceptors are preserved in wi/wi mutant mice. $\boldsymbol{A}$, Expression of Rx3 and Whrn in DRG of adult ( $>6$ months) wild-type and wi/wi mutant mice. $\boldsymbol{B}$, Numbers of Rx3 ${ }^{+}$neurons in adult L2 DRG in wild-type and wi/wi mutants are $\operatorname{similar}(p=$ $0.389, t$ test). C, pSN axon collaterals as visualized by expression of Pv in p15 wild-type and wi/wi mutant mice. $\boldsymbol{i}^{\prime}, \boldsymbol{i}^{\prime \prime}, \boldsymbol{i}^{\prime}, \boldsymbol{i i}^{\prime \prime}$ Enlargements of ventral motor neuron areas. D, vGluT1 ${ }^{+}$synaptic terminals oppose Shank1a postsynaptic densities in p15 
A

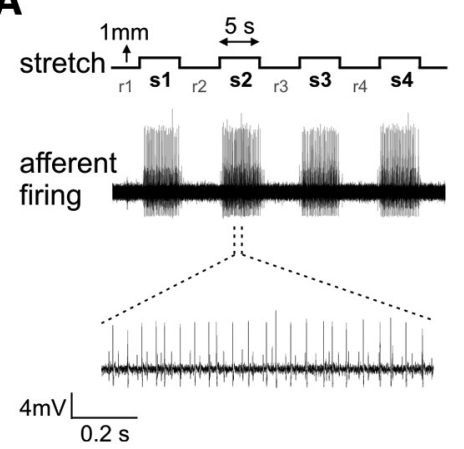

B

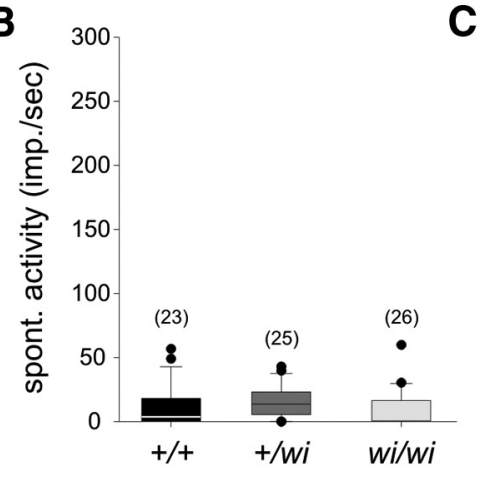

C

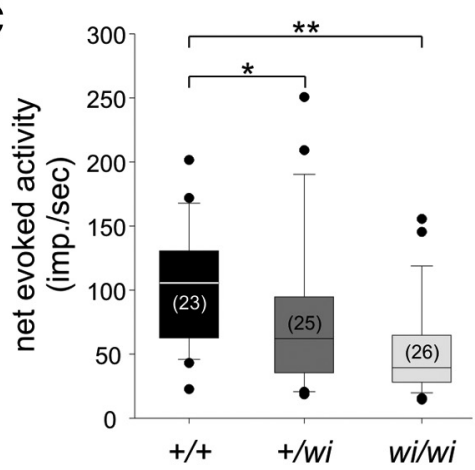

Figure 4. Soleus muscle stretch-evoked responses are reduced in wi/wi mutant mice. $\boldsymbol{A}$, Experimental paradigm of Soleus (Sol) muscle nerve extracellular recordings. Top, Sequence of muscle stretches $(1 \mathrm{~mm})$ to which Sol muscles were subjected during a single trial. Stretch episodes (s1-s4) were alternated with intervals during which muscles were kept at resting length ( $\mathrm{r} 1$-r4) (for details, see Materials and Methods). Middle, Recording of firing activity of wild-type Sol muscle nerve during a stretch trial. Bottom, High-resolution image of pSN firing activity during a $1 \mathrm{~s}$ stretch

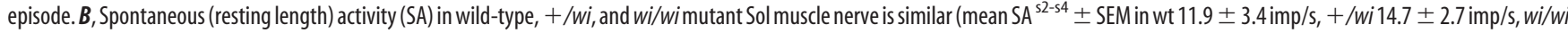
$9.2 \pm 2.8 \mathrm{imp} / \mathrm{s} ;$ wt vs $+/$ wi, $p=0.22$; wt vs wi/wi, $p=0.32$, Mann-Whitney rank sum test). C, Net evoked activity (NEA) in Sol muscle nerve in wild-type, $+/$ wi, and wi/wi mutant mice.

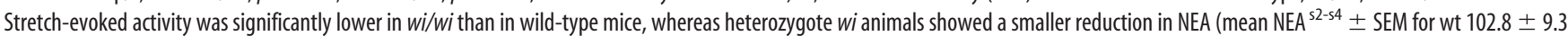
$\mathrm{imp} / \mathrm{s},+/$ wi $77 \pm 12.9 \mathrm{imp} / \mathrm{s}$, wi/wi $51.6 \pm 7.0 \mathrm{imp} / \mathrm{s}$; wt vs $+/$ wi, $p=0.019 ;$ wt vs wi/wi, $p<0.001$, Mann-Whitney rank sum test). $\boldsymbol{B}, \boldsymbol{C}$, Tails are extended to the largest and smallest data points, which are not outliers. Black dots represent outliers. Number of Sol muscles analyzed is indicated in parentheses. ${ }^{*} p<0.05,{ }^{* *} p<0.01$.

ity and function, a possibility suggested by recent reports that Whrn contributes to the paranodal organization of myelinated axons (Green et al., 2013).

To address this issue, we asked whether Whrn regulates pSN action potential conductance or sensory-motor synaptic connectivity in an isolated spinal cord-hindlimb preparation (Fig. 5A) (Shneider et al., 2009; Mentis et al., 2011). We found that, at p12-13, pSNs in wild-type and wi/wi mutant mice had similar conduction velocities (mean \pm SEM $4.7 \pm 0.5 \mathrm{~ms}$ for wt and $4.0 \pm 0.5 \mathrm{~ms}$ for $w i / w i, p=0.2$, Mann-Whitney rank sum test) (Fig. $5 B, C$ ). Likewise, when we examined motor neuron compound action potentials following stimulation of L3 dorsal roots, we observed no differences in either the latency or amplitude of the evoked motor neuron responses between wild-type and wi/wi mutants (Fig. 5D-F). Last, we measured the pSN-induced $\mathrm{H}$-reflex, which assesses the entire MS-afferent monosynaptic stretch-reflex circuit (Fig. $5 G$ ) but again detected no difference in the H-reflex latency between wild-type and wi/wi mutants (Fig. $5 H, I$ ).

Thus, the absence of Whrn protein does not impair pSN action potential propagation, or sensory-motor synaptic transmission. These findings support the idea that Whrn is required for mechanosensory signaling in peripheral pSN endings.

\section{Whrn is not required for the glutamate-mediated sensitization of MS sensory endings}

We also explored how Whrn might contribute to proprioceptor firing frequency, examining whether Whrn serves a role in the glutamate-mediated sensitization of pSN sensory endings. Glutamate released from sensory terminals is thought to act through atypical phospholipase-D-coupled glutamate receptors to augment MS-afferent sensitivity to stretch (Fig. 6A, B) (Bewick et al., 2005). We therefore determined whether the reduced stretchevoked activity in wi/wi mutants is a consequence of impairments in this glutamate-mediated sensitization of $\mathrm{pSN}$ sensory endings.

To test this, we assessed the ability of exogenously provided glutamate to augment afferent discharge frequency in wild-type and wi/wi mutant mice. For wild-type Sol pSNs, we found that glutamate $(10 \mathrm{nM}, 100 \mathrm{~nm}$, or $1 \mu \mathrm{M})$ did not increase spontaneous discharge activity (Fig. 6C). However, glutamate resulted in significant increases in stretch-evoked activity, on average reaching an increase of $53 \pm 18 \%$ of baseline levels at $1 \mu \mathrm{M}$ glutamate in wild-type pSNs (Fig. $6 B, D, E$ ), consistent with previous findings (Bewick et al., 2005).

The ability of glutamate to increase afferent excitability was preserved in wi/wi mutant pSNs (Fig. $6 B, D, E$ ). In the presence of glutamate, mutant Sol pSNs exhibited a dose-dependent increase in afferent firing (Fig. 6D). At each concentration, the glutamatemediated increase in stretch-evoked firing in wi/wi Sol afferents was proportionally similar to that observed for wild-type Sol afferents (Fig. 6E). Although glutamate could offset the reduced stretch-evoked firing in wi/wi mutant Sol afferents, sensory discharge frequencies remained below those observed in wild-type (Fig. 6D). Based on these results, we infer that wi/wi mutant pSNs remain equally responsive to the activity of exogenously provided glutamate. This finding suggests that glutamate and Whrn operate through independent pathways to regulate afferent discharge levels in response to muscle stretch.

\section{Whrn is required for the fidelity of $\mathrm{pSN}$ firing in response to repeated stretch}

To test whether Whrn controls the fidelity of pSN firing, we assessed the variability and constancy of Sol afferent firing rates in wild-type and wi/wi mutant mice. We compared the variability of wild-type and wi/wi mutant Sol pSN firing rates during stretch and calculated the coefficient of variation (CV) across $100 \mathrm{~ms}$ bins of each stretch episode (Fig. 7A). For wild-type mice, the CV was $0.16 \pm 0.001$ (Fig. $7 B$ ), but for wi/wi mutants the variability in firing rates was much larger, with a CV of $0.24 \pm 0.02(p<$ 0.001, Mann-Whitney rank sum test) (Fig. 7B). Thus, during stretch, the responses of wi/wi mutant Sol pSNs show considerably more variability in their sustained firing level.

We next examined the constancy in afferent firing across the trials (separated by $\sim 15$ min of rest) to which wild-type and wi/wi mutant Sol muscles were subjected (Fig. 7A). However, when we compared the mean afferent firing rates of trial 1 and trial 3, we observed no intertrial difference for either wild-type or wi/wi mutants (wt t 1 mean NEA ${ }^{\mathrm{s} 2-\mathrm{s} 4}$ vs $\mathrm{t} 3$ mean $^{\mathrm{NEA}}{ }^{\mathrm{s} 2-\mathrm{s} 4} p=0.81$, wi/witl mean NEA ${ }^{\mathrm{s} 2-\mathrm{s} 4}$ vs $\mathrm{t} 3$ mean $\mathrm{NEA}^{\mathrm{s} 2-\mathrm{s} 4} p=0.83$; Wilcoxon signed rank test) (Fig. 7C). We also assessed the intratrial afferent firing rates, comparing stretch episodes 2 and 4 of each trial and found that wild-type and wi/wi mutant pSNs showed a similar 
A

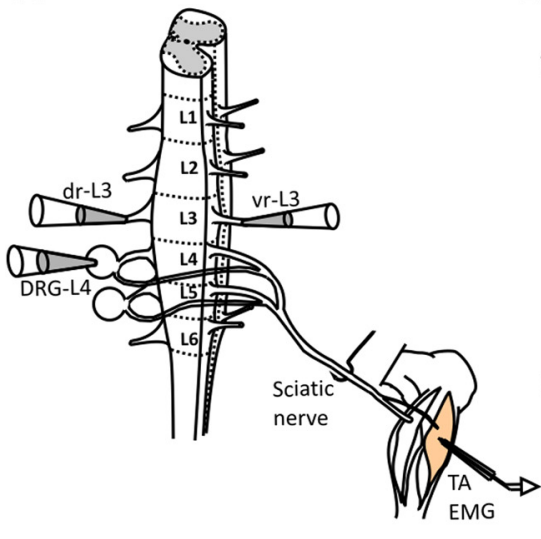

B

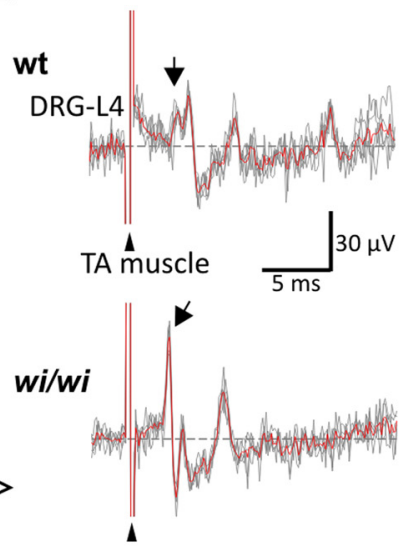

C

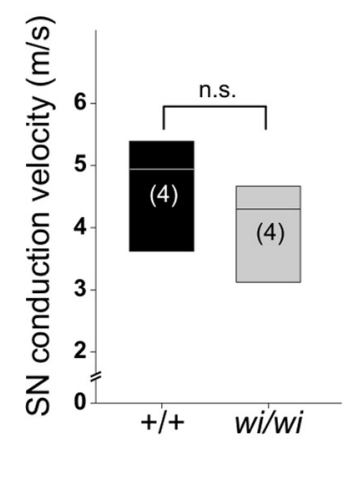

$\mathbf{F}$

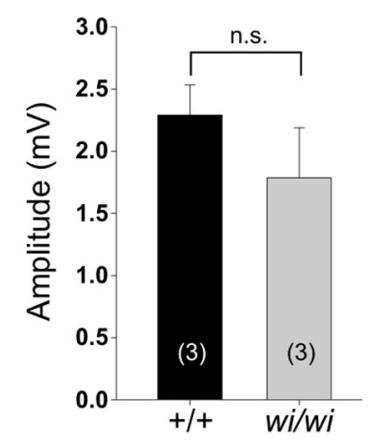

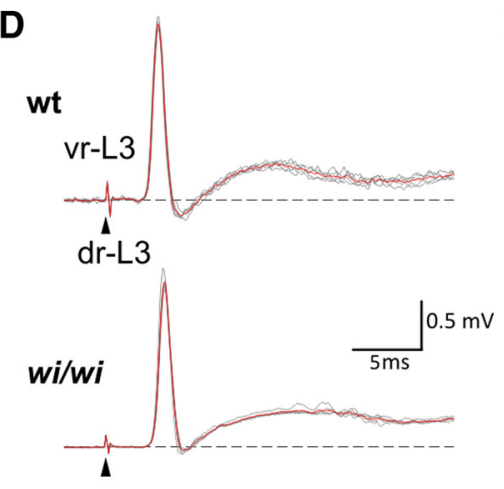

E
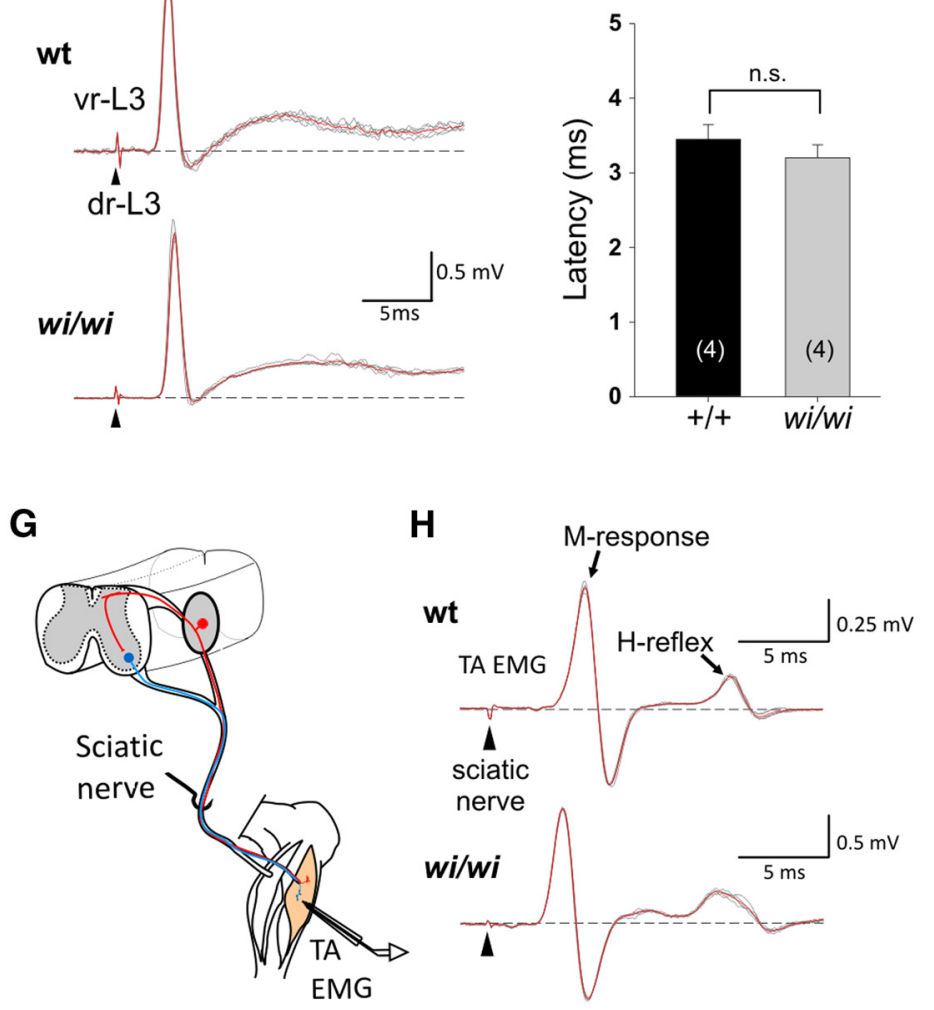

I

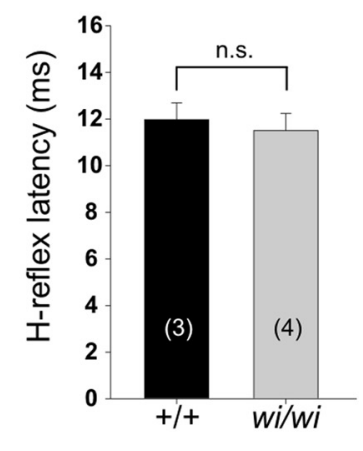

Figure 5. Normal pSN axonal conduction velocity and sensory-motor connectivity in wi/wi mutant mice. $\boldsymbol{A}$, Schematic of the isolated spinal cord-hindlimb preparation used in conduction velocity and sensory-motor connectivity assays. Stimulated nerves and muscles as well as recording sites are indicated. L1-6, Lumbar segment L1-L6; dr-L3, dorsal root-L3; vr-L3, ventral root-L3; TA-EMG, tibialis anterior-electromyogram. $\boldsymbol{B}$, Recordings from L 4 DRG following stimulation of TA muscle in wild-type and wi/wi mutant mice. Arrowheads indicate time of TA stimulation; arrows indicate time point at which the earliest responses are detected in $\angle 4$ DRG. C, Sensory axon conduction velocity in wild-type and wi/wi mutant mice measured from recordings described in $\boldsymbol{B}$ are not significantly different (mean \pm SEM wt $4.7 \pm 0.5 \mathrm{~m} / \mathrm{s}$, wi/wi $4.0 \pm 0.5 \mathrm{~m} / \mathrm{s} ; p=0.2$, Mann-Whitney rank sum test). $D$, vr-L3 compound motor neuron (MN) responses following stimulation of $\mathrm{dr}$-L3 in wild-type and wi/wi mutant mice. Arrowheads indicate time of stimulation. $E$, Latencies of the initial $\mathrm{L} 3 \mathrm{MN}$ responses following dr-L3 stimulation are similar in wild-type and wi/wi mutant mice (wt $3.5 \pm 0.2 \mathrm{~ms}$; wi/wi $3.2 \pm 0.2 \mathrm{~ms} ; p=0.37$, $t$ test). $F$, Upon stimulation of dr-L3, the amplitude of the maximum compound action potential in vr-L3 in wi/wi mutant mice is similar to that in wild-type (wt $2.3 \pm 0.2 \mathrm{mV}$; wi/wi $1.8 \pm 0.4 \mathrm{mV} ; p=0.35, t$ test). G, Schematic of the monosynaptic stretch reflex circuit assessed in H-reflex recordings. Red neuron represents a proprioceptive sensory neuron. Blue neuron represents a motor neuron. $\boldsymbol{H}$, EMG recordings of TA muscle following stimulation of the sciatic nerve in wild-type and wi/wi mutant mice. Peaks corresponding to M-response and $\mathrm{H}$-response are indicated. I, Latency measurements of the H-reflex are similar in wild-type and wi/wi mutant mice (wt $12.0 \pm 0.7 \mathrm{~ms} ;$ wi/wi $11.5 \pm 0.7 \mathrm{~ms} ; p=0.68, t$ test). Error bars indicate SEM. $\boldsymbol{C}, \boldsymbol{E}, \boldsymbol{F}, \boldsymbol{H}$, The number of animals analyzed is indicated in parentheses. n.s., Not significant.

decline in afferent firing across trial episodes s2-s4 (wt $3.3 \pm$ $2.0 \%$; wi/wi $4.8 \pm 3.4 \%$ ) (Fig. 7D). However, when we included the first stretch episode of each trial in our analysis (s1; which follows after an extended rest period), we find a much larger intratrial difference in wi/wi mutants, but not in wild type. For wild-type Sol pSNs, the rate of afferent firing in s1 was similar to the mean firing rate of $s 2-s 4$ ( $p=0.77$, paired $t$ test) (Fig. $7 E, F$ ).
Instead, for wi/wi mutant Sol pSNs, we measured a $12 \pm 3 \%$ decline in afferent firing when comparing $s 1$ and $s 2-s 4(p=$ 0.003, Wilcoxon signed rank test) (Fig. $7 E, F)$. We observed a similar trend when comparing $s 1$ to stretch episodes s2, s3, and s4 individually (for wt: Friedman repeated measures ANOVA on ranks, $p=0.013$; post hoc Tukey test with $p<0.05$ only for s2 vs s4; for wi/wi: Friedman repeated measures ANOVA on ranks, $p<$ 
A
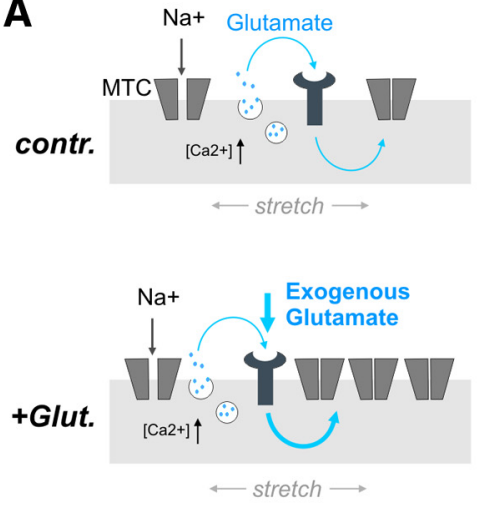

C

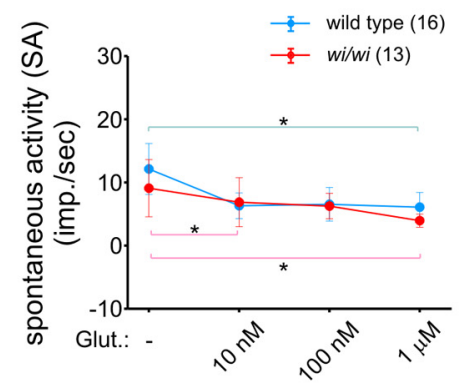

B
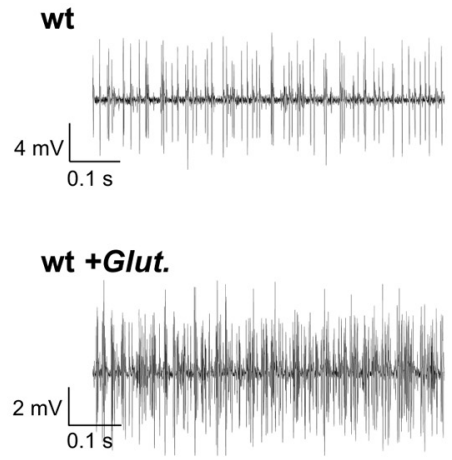

D

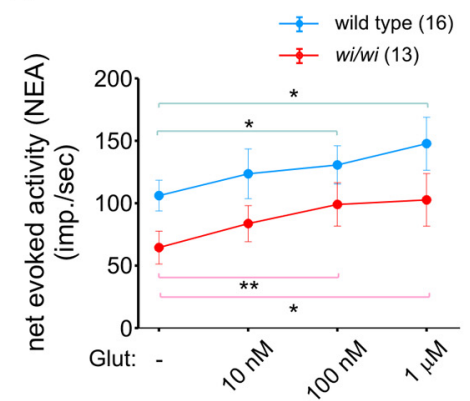

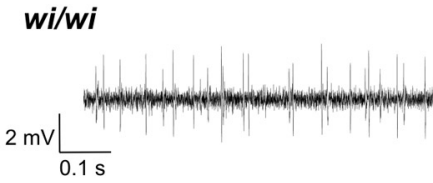

wi/wi +Glut.

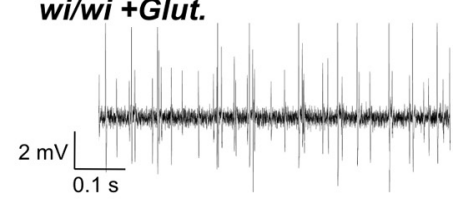

E

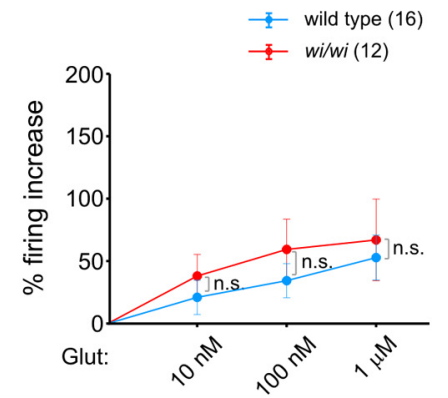

Figure 6. Whrn facilitates $\mathrm{pSN}$ stretch-evoked activity independently from a glutamate-based mechanism of $\mathrm{pSN}$ sensitization to muscle stretch. $A$, Influence of glutamate on wild-type and wi/wi mutant Sol pSN firing rates. Schematics of pSN sensory endings illustrating that endogenous (control) or exogenous glutamate ( + Glut.) increases pSN sensitivity to stretch (illustrated by an increase in the number of stretch-sensitive mechanotransduction channels [MTC]; the actual mechanism has yet to be determined). $\boldsymbol{B}$, Representative recordings of wild-type and wi/wi mutant Sol muscles nerves in the absence (top) and presence of $10 \mathrm{~nm}$ glutamate (bottom). C, Quantification of spontaneous discharge activity in wild-type (blue) and wi/wi mutant (red) Sol pSNs in the presence of increasing concentrations of glutamate. Rather than increasing pSN baseline excitability, glutamate appears to dampen spontaneous activity levels. D, NEA in pSNs of wild-type (blue) and wi/wi mutant (red) Sol muscles in the presence of increasing amounts of glutamate. In both wild-type and wi/wi mutants, glutamate results in an increased afferent firing rate (mean NEA \pm SEM without glut, $10 \mathrm{~nm}$ glut, $100 \mathrm{~nm}$ glut, $1 \mu \mathrm{m}$ glut for wt: $106.0 \pm 12.4 \mathrm{imp} / \mathrm{s}, 123.5 \pm 20 \mathrm{imp} / \mathrm{s}, 130.6 \pm 15.3 \mathrm{imp} / \mathrm{s}, 147.7 \pm 17.9 \mathrm{imp} / \mathrm{s} ;$ for wi $/$ wi: $64.4 \pm 13.1 \mathrm{imp} / \mathrm{s}, 83.6 \pm 14.5 \mathrm{imp} / \mathrm{s}, 99.0 \pm 17.3 \mathrm{imp} / \mathrm{s}$, $102.6 \pm 21.1 \mathrm{imp} / \mathrm{s}$; wt without glut vs $100 \mathrm{~nm}, p=0.026$; wt without glut vs $1 \mu \mathrm{m}, p=0.034$; wi/wi without glut vs $100 \mathrm{~nm}, p=0.009$; wi/wi without glut vs $1 \mu \mathrm{m}, p=0.045$, paired $t$ test). $E$, Percentage increase in pSN firing in the presence of glutamate, for wild-type (blue) and wi/wi mutant (red) Sol pSNs. Increases in pSN firing are proportionally similar between wild-type and wi/wi mutants (wt vs wi/wi in $10 \mathrm{~nm}$ glut, $p=0.39$; in $100 \mathrm{~nm}$ glut, $p=0.69$; in $1 \mu \mathrm{m}$ glut, $p=0.91$; Mann-Whitney rank sum test). C $E$, Error bars indicate SEM. Number of Sol muscles analyzed is indicated in parentheses. ${ }^{*} p<0.05,{ }^{* *} p<0.01$. n.s., Not significant.

0.001; post hoc Tukey test, s1 vs s2: $q=2.7, p>0.05$; 1 vs $\mathrm{s} 3: q=$ $7.5, p<0.05$; s1 vs s4: $q=7.6, p<0.05)$. Thus, in wi/wi mutants, Sol pSN stretch-evoked responses fatigue more easily, resulting in a reduced fidelity in their response to repeated stimulation.

In summary, these findings indicate that proprioceptor stretchevoked responses are diminished and irregular in wi/wi mutant mice, and support the idea that Whrn facilitates the coding of mechanical stretch to achieve appropriate levels of afferent firing.

\section{Discussion}

Mechanical force activates sensory nerve terminals by opening mechanosensitive transduction channels, eliciting receptor generator potentials, and initiating action potentials. Efforts to elucidate the basis of these sensory processes have identified several candidate transduction channel molecules but have yielded little insight into the mechanism by which distinct mechanoreceptive modalities acquire their gating properties or sensory coding mechanisms. Our analysis of the role of the PDZ-scaffold protein Whirlin in proprioceptor sensory signaling provides evidence that Whrn plays an integral part in regulating pSN mechanosensory coding efficacy. The activity of this adaptor molecule in proprioceptor and vestibulocochlear hair cell signaling raises the possibility that accessory transduction molecules confer com- mon features of sensory processing to different mechanosensory systems.

\section{Whirlin function in mechanosensory coding}

Proprioceptors exhibit a marked reduction in stretch-evoked firing in wi/wi mutants and show a reduced fidelity in response to repeated stretch stimuli. Nevertheless, stretch-evoked discharge activity in wi/wi mutant pSNs is not completely lost. We also find that wi/wi mutant pSNs retained their sensitivity to glutamate to an extent similar to that observed for wild-type pSNs. This implies that the glutamate-dependent sensitization pathway does not depend on Whrn, and suggests that the two pathways operate in parallel to regulate pSN firing. Thus, essential components of the transduction machinery appear to be active in wi/wi mutants, but mechanical stimuli are inefficiently translated into action potentials.

The reduction in stretch-evoked impulse activity in wi/wi mutant mice appears not to be the consequence of a loss or overt morphological abnormalities in the sensory terminals of these afferents. Nor did we observe a decrease in their axonal conduction velocity. This suggests that Whrn functions in the sensory coding process: between the moment of mechanical stimulation and the initiation of action potentials within the stretch-sensitive afferent terminal. pSN afferent spike frequency is directly pro- 
A

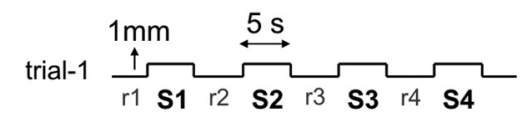

trial-2

trial-3

\section{D}

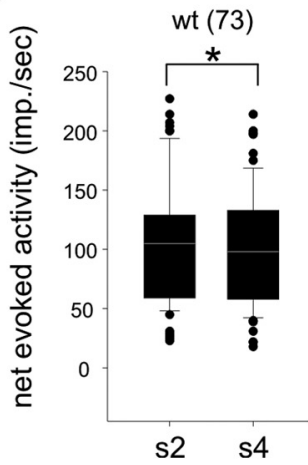

B

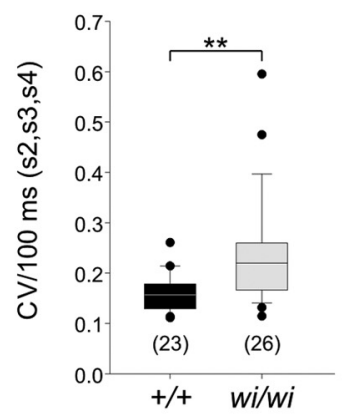

E
C
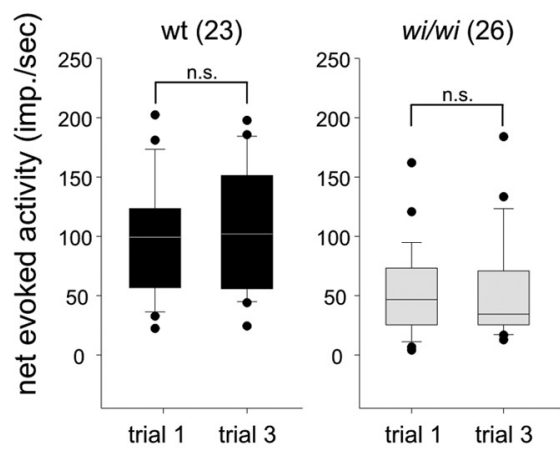

$\mathbf{F}$
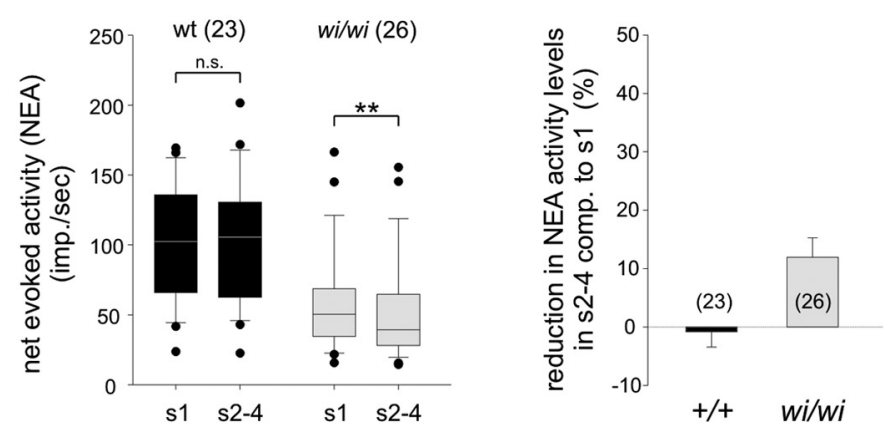

Figure 7. pSNs in wi/wi mutants exhibit decreased fidelity in response to repeated muscle stretch. $A$, Diagram of stretch paradigm used in experimental soleus muscle nerve recordings. $B$, CV across $100 \mathrm{~ms}$ bins of stretch episodes S2-S4 for wild-type $\left(0.16 \pm 1 \mathrm{e}^{-3}\right)$ and wi/wi mutant $(0.24 \pm 0.02)$ Sol pSN firing rates differ significantly $(p<0.001$, Mann-Whitney rank sum test). $\boldsymbol{C}$, Comparisons of net stretch-evoked firing rates between trials 1 and 3 , in wild-type and wi/wi mutant Sol pSNs. For both wild-type and wi/wi mutants, Sol muscle nerve discharge was similar across trials (wt $p=0.81$, wi/wip $=0.83$, Wilcoxon signed rank test). $\boldsymbol{D}$, Comparisons of net stretch-evoked firing rates between stretch epochs s2 and s4, in wild-type and wi/wi mutant Sol pSNs. In both wild-type and wi/wi mutants, stretch-evoked activity levels in $s 4$ are reduced compared with $s 2$ (wt $s 2$ vs $s 4, p=0.015$; wi/wi s2 vs $s 4, p<0.001$; Wilcoxon signed rank test). $E$, Comparisons of $p S N$ firing rates during stretch episode 1 ( preceded by extended rest) and episodes $s 2-s 4$, for wild-type (black) and wi/wi mutant (gray) Sol pSNs. Firing rates are similar for wild-type (mean NEA \pm SEM for wt s1 $101.9 \pm 8.7 \mathrm{imp} / \mathrm{s}$, wt s2-s4 $102.8 \pm 9.3 \mathrm{imp} / \mathrm{s}, p=0.77$, paired $t$ test; for wi $/$ wi $158.6 \pm 7.2 \mathrm{imp} / \mathrm{s}$, wi/wi s2-s4 $51.6 \pm 7.0 \mathrm{imp} / \mathrm{s}, p=0.003$, Wilcoxon signed rank test). $F$, Decline in NEA activity level, comparing stretch episode 11 and episodes $s 2-s 4$, for wild-type ( $0.8 \pm 2.6 \%$; black) and wi/wi mutant (11.9 $\pm 3.4 \%$; gray) Sol pSNs. $\boldsymbol{B}-\boldsymbol{E}$, Tails are extended to the largest and smallest data points which are not outliers. Black dots indicate outliers. $\boldsymbol{F}$, Error bar indicates SEM. Number of Sol muscles analyzed $(\boldsymbol{B}, \boldsymbol{C}, \boldsymbol{E}, \boldsymbol{F})$ or stretch epochs $(\boldsymbol{D})$ are indicated in parentheses. ${ }^{*} p<0.05,{ }^{* *} p<0.01$. n.s., Not significant.

portional to the receptor generator potential (Hunt and Ottoson, 1975), which in turn is influenced by the number and type of transduction channels expressed, by their resting potential, and by their adaptive properties. It is unclear whether Whrn contributes to the regulation of one or more of these functional features. PDZ-scaffold proteins recruit macromolecular complexes to specific subcellular localizations (Kim and Sheng, 2004). As such, Whrn could localize the mechanosensitive transduction channel to sensory terminals or could be necessary for the recruitment or clustering of auxiliary transduction channel proteins that regulate channel gating or conductance (Goodman et al., 2002; Jepson et al., 2012; Lapatsina et al., 2012; Xiong et al., 2012). Alternatively, Whrn could facilitate the stretch-induced response by organizing cytoskeletal components that regulate the membrane tension of proprioceptor sensory endings (Delprat et al., 2005; Prost et al., 2007).

What are the implications of reduced pSN activity for sensorymotor control? MS afferents signal muscle length through their firing rate (Houk et al., 1966; Hunt and Ottoson, 1975). This implies that the reduced pSN firing in response to an increase in muscle length in wi/wi mutant mice may skew the relationship between mechanical input and sensory output. However, it is possible that in mice raised with reduced afferent spike activity, coding of muscle length will adjust centrally according to this lower $\mathrm{PSN}$ spike frequency. The lack in constancy in pSN output in response to repetitive stimulation in Whrn mutants could cause a more debilitating effect on motor control, however. In particular, during corrective reflex responses that bypass central control, mismatches between the level of spindle activation and their sensory output could result in a reduced ability to generate the appropriate corrective motor response. The overt vestibular dysfunction in wi/wi mutant mice precluded an assessment of any sensory-motor behavioral phenotypes (Holme et al., 2002, Mburu et al., 2003). Assessing how the loss of Whrn affects sensory-motor behavior will likely require selective and acute elimination of Whrn protein from pSNs.

\section{The selectivity of Whirlin expression in proprioceptive muscle afferents}

The selectivity of Whrn expression in proprioceptive sensory neurons suggests that the protein confers a specific feature of the mechanosensory signaling mechanism. DRG contain numerous classes of large-caliber low-threshold cutaneous mechanoreceptive neurons, each with distinct activation and adaptation properties and specialized to convey a different aspect of discriminative touch (Johnson, 2001; Abraira and Ginty, 2013). Pacinian and Meissner afferents are both rapidly adapting but differ in their frequency selectivity (Talbot et al., 1968; Johnson, 2001). In contrast, Merkel-cell afferents, like proprioceptors, are slowly adapting (Hunt and Ottoson, 1975; Wellnitz et al., 2010). Whirlin is not expressed in these other classes of somatic mechanoreceptors, suggesting that distinct mechanoreceptor subclasses engage dif- 
ferent accessory molecules to confer the modality-specific features of mechanosensory coding.

Whrn expression marks all three classes of pSNs: Group Ia and Group II MS, as well as Group Ib GTO, afferents. This observation suggests that these three proprioceptive subclasses rely on mechanosensory-encoding mechanisms that are similar, despite the distinct morphological features of their sensory receptive endings (MS and GTO) and their individual physiological properties (Hunt and Ottoson, 1975; Fukami and Wilkinson, 1977; Jami, 1992; Banks et al., 2009). Consistent with this idea, we find that Whirlin is required to regulate pSN discharge activity in response to static muscle stretch, indicating a role for Whirlin in both classes of MS afferents. It is unclear whether GTO afferents also depend on Whirlin to enhance their responses to muscle contraction.

\section{Common and divergent effectors in mechanosensory processing}

The loss of Whrn does not result in developmental abnormalities or in the loss of proprioceptors but selectively alters their mechanosensory response to stretch. This contrasts with Whrn mutant hair cells and photoreceptors, which exhibit morphological abnormalities and degenerate (Holme et al., 2002; Maerker et al., 2008; Yang et al., 2010). The discrepancy in Whrn dependency could have its basis in the various different Whrn proteininteraction domains (Fig. $2 F$ ), which appear to support different cellular activities in different tissues (Mburu et al., 2003; Yang et al., 2010). Photoreceptors critically depend on the N-terminal PDZ-domains of the full-length Whrn protein, whereas the C-terminal proline-rich and PDZ domain appears dispensable for retinal function. In contrast, whereas the C-terminal domains are essential for cochlear and vestibular hair cell development and function, mutations in the N-terminal PDZ-domains only affect the morphology of outer hair cells in the cochlea (Yang et al., 2010). Consequently, different Whrn N- or C-terminal isoforms containing only one or two of the functional domains can, depending on the tissue, partially compensate for the loss of the full-length Whrn protein (Mburu et al., 2003; Yang et al., 2010). Thus, although we were not able to detect the expression of the dominant short C-terminal Whrn isoform, the maintenance of proprioceptors in $\mathrm{Whrn}^{\text {wi/wi }}$ mutants could reflect the residual activity of the truncated $\mathrm{N}$-terminal region or the existence of a short N-terminal isoform (Mburu et al., 2003). However, no apparent behavioral defects were observed in Whrn mutant mice lacking the N-terminal PDZ domains of the Whrn protein (Whrn ${ }^{\triangle P D Z 1,2}$; Yang et al., 2010). Thus, although the pSN response to stretch in these mutants may be similarly compromised as in $W h r n^{w i / w i}$ mutants, the absence of overt motor defects suggests that proprioceptors are most likely preserved in these mutants.

The difference in the requirement for the Whrn-protein in hair cells, photoreceptors, and proprioceptors alternatively could reflect that the various biological functions of Whrn are only partially shared across these tissues. In support of this idea, in preliminary expression studies in proprioceptors, we failed to detect expression of many key Whrn-associated molecules that previously were identified in hair cells and photoreceptors, including Usherin, Very Large G-protein-coupled Receptor 1 (VLRG1), SANS, and Myosin 15a (Kremer et al., 2006) (J.C.d.N. and T.M.J., unpublished results). Known Whrn partners that we did observe in our proprioceptor-enriched population were Myosin 7a, NGL1 (independently identified in our pSN screen; Fig 1C), Cask, and Mpp1/p55 (J.C.d.N. and T.M.J., unpublished results). These data begin to suggest that the Whrn macromolecular complex involved in mechanosensory signaling in pSNs, and possibly hair cells, may constitute a different molecular complex serving a separate biological activity.

The activity of Whirlin in MS afferents and hair cells raises the issue of the parallels between proprioceptor and hair cell mechanoreceptive signaling. MS afferents lack a dedicated transduction cell, but the response properties of the two sensory systems share several features. Both sensory receptor classes exhibit dynamic (rapidly adapting) responses at the onset of stimulus activation (muscle stretch/hair cell stereocilia deflection) (Hunt and Ottoson, 1975; Kennedy et al., 2003). MS afferents also exhibit slow adaptation kinetics during static activity, maintaining firing in the presence of a continued stimulus, a feature that is observed in hair cells (Hunt and Ottoson, 1975; Gillespie and Corey, 1997; Fettiplace et al., 2001). And both sensory systems exhibit tonic activity, implying that the resting membrane potential is partly depolarized in the absence of mechanical stimulation and so contributes to their extremely rapid activation kinetics (Matthews, 1972; Safieddine et al., 2012). Whether these response properties rely on a common, and perhaps, a Whrn-directed, mechanism awaits a more thorough understanding of the molecular characteristics that define proprioceptor sensory processing.

\section{References}

Abraira VE, Ginty DD (2013) The sensory neurons of touch. Neuron 79: 618-639. CrossRef Medline

Allen Institute for Brain Science (2012) Allen Spinal Cord Atlas http://mousespinal.brain-map.org/.

Banks RW, Hulliger M, Saed HH, Stacey MJ (2009) A comparative analysis of the encapsulated end-organs of mammalian skeletal muscles and of their sensory nerve endings. J Anat 214:859-887. CrossRef Medline

Betley JN, Wright CV, Kawaguchi Y, Erdélyi F, Szabó, G, Jessell TM, Kaltschmidt JA (2009) Stringent specificity in the construction of a GABAergic presynaptic inhibitory circuit. Cell 139:161-174. CrossRef Medline

Bewick GS, Reid B, Richardson C, Banks RW (2005) Autogenic modulation of mechanoreceptor excitability by glutamate release from synaptic-like vesicles: evidence from the rat muscle spindle primary sensory ending. J Physiol 562:381-394. CrossRef Medline

Buffelli M, Burgess RW, Feng G, Lobe CG, Lichtman JW, Sanes JR (2003) Genetic evidence that relative synaptic efficacy biases the outcome of synaptic competition. Nature 424:430-434. CrossRef Medline

Chen AI, de Nooij JC, Jessell TM (2006) Graded activity of transcription factor Runx3 specifies the laminar termination pattern of sensory axons in the developing spinal cord. Neuron 49:395-408. CrossRef Medline

Chen CC, Wong CW (2013) Neurosensory mechanotransduction through acid-sensing ion channels. J Cell Mol Med 17:337-349. CrossRef Medline

Coste B, Mathur J, Schmidt M, Earley TJ, Ranade S, Petrus MJ, Dubin AE, Patapoutian A (2010) Piezol and Piezo2 are essential components of distinct mechanically activated cation channels. Science 330:55-60. CrossRef Medline

Del Valle ME, Cobo T, Cobo JL, Vega JA (2012) Mechanosensory neurons, cutaneous mechanoreceptors, and putative mechanoproteins. Microsc Res Tech 75:1033-1043. CrossRef Medline

Delprat B, Michel V, Goodyear R, Yamasaki Y, Michalski N, El-Amraoui A, Perfettini I, Legrain P, Richardson G, Hardelin JP, Petit C (2005) Myosin XVa and whirlin, two deafness gene products required for hair bundle growth, are located at the stereocilia tips and interact directly. Hum Mol Genet 14:401-410. CrossRef Medline

Demireva EY, Shapiro LS, Jessell TM, Zampieri N (2011) Motor neuron position and topographic order imposed by $\beta$ - and $\gamma$-catenin activities. Cell 147:641-652. CrossRef Medline

de Nooij JC, Doobar S, Jessell TM (2013) Etv1 inactivation reveals proprioceptor subclasses that reflect the level of NT3 expression in muscle targets. Neuron 77:1055-1068. CrossRef Medline

Edgar R, Domrachev M, Lash AE (2002) Gene Expression Omnibus: NCBI gene expression and hybridization array data repository. Nucleic Acids Res 30:207-210. CrossRef Medline 
Fettiplace R, Ricci AJ, Hackney CM (2001) Clues to the cochlear amplifier from the turtle ear. Trends Neurosci 24:169-175. CrossRef Medline

Fukami Y, Wilkinson RS (1977) Responses of isolated Golgi tendon organs of the cat. J Physiol 265:673-689. CrossRef Medline

Gillespie PG, Corey DP (1997) Myosin and adaptation by hair cells. Neuron 19:955-958. CrossRef Medline

Gillespie PG, Müller U (2009) Mechanotransduction by hair cells: models, molecules, and mechanisms. Cell 139:33-44. CrossRef Medline

Goodman MB, Ernstrom GG, Chelur DS, O'Hagan R, Yao CA, Chalfie M (2002) MEC-2 regulates C. elegans DEG/ENaC channels needed for mechanosensation. Nature 415:1039-1042. CrossRef Medline

Green JA, Yang J, Grati M, Kachar B, Bhat MA (2013) Whirlin, a cytoskeletal scaffolding protein, stabilizes the paranodal region and axonal cytoskeleton in myelinated axons. BMC Neurosci 14:96. CrossRef Medline

Hippenmeyer S, Vrieseling E, Sigrist M, Portmann T, Laengle C, Ladle DR, Arber S (2005) A developmental switch in the response of DRG neurons to ETS transcription factor signaling. PLoS Biol 3:878-890. CrossRef Medline

Holme RH, Kiernan BW, Brown SD, Steel KP (2002) Elongation of hair cell stereocilia is defective in the mouse mutant whirler. J Comp Neurol 450: 94-102. CrossRef Medline

Houk JC, Cornew RW, Stark L (1966) A model of adaptation in amphibian spindle receptors. J Theor Biol 12:196-215. CrossRef Medline

Hunt CC, Ottoson D (1975) Impulse activity and receptor potential of primary and secondary endings of isolated mammalian muscle spindles. J Physiol 252:259-281. CrossRef Medline

Hunt CC, Wilkinson RS, Fukami Y (1978) Ionic basis of the receptor potential in primary endings of mammalian muscle spindles. J Gen Physiol 71:683-698. CrossRef Medline

Jami L (1992) Golgi tendon organs in mammalian skeletal muscle: functional properties and central actions. Physiol Rev 72:623-666. Medline

Jepson JE, Shahidullah M, Lamaze A, Peterson D, Pan H, Koh K (2012) dyschronic, a Drosophila homolog of a deaf-blindness gene, regulates circadian output and Slowpoke channels. PLoS Genet 8:e1002671. CrossRef Medline

Johnson KO (2001) The roles and functions of cutaneous mechanoreceptors. Curr Opin Neurobiol 11:455-461. CrossRef Medline

Kennedy HJ, Evans MG, Crawford AC, Fettiplace R (2003) Fast adaptation of mechanoelectrical transducer channels in mammalian cochlear hair cells. Nat Neurosci 6:832-836. CrossRef Medline

Kim E, Sheng M (2004) PDZ domain proteins of synapses. Nat Rev Neurosci 5:771-781. CrossRef Medline

Kramer I, Sigrist M, de Nooij JC, Taniuchi I, Jessell TM, Arber S (2006) A role for Runx transcription factor signaling in dorsal root ganglion sensory neuron diversification. Neuron 49:379-393. CrossRef Medline

Kremer H, van Wijk E, Märker T, Wolfrum U, Roepman R (2006) Usher syndrome: molecular links of pathogenesis, proteins and pathways. Hum Mol Genet 15:R262-R270. CrossRef Medline

Lapatsina L, Brand J, Poole K, Daumke O, Lewin GR (2012) Stomatindomain proteins. Eur J Cell Biol 91:240-245. CrossRef Medline

Liley AW (1956) An investigation of spontaneous activity at the neuromuscular junction of the rat. J Physiol 132:650-666. CrossRef Medline

Maerker T, van Wijk E, Overlack N, Kersten FF, McGee J, Goldmann T, Sehn E, Roepman R, Walsh EJ, Kremer H, Wolfrum U (2008) A novel Usher protein network at the periciliary reloading point between molecular transport machineries in vertebrate photoreceptor cells. Hum Mol Genet 17:71-86. CrossRef Medline

Malin SA, Davis BM, Molliver DC (2007) Production of dissociated sensory neuron cultures and considerations for their use in studying neuronal function and plasticity. Nat Protoc 2:152-160. CrossRef Medline

Matthews PBC (1972) Mammalian muscle receptors and their central actions. London: Edward Arnold.
Mburu P, Mustapha M, Varela A, Weil D, El-Amraoui A, Holme RH, Rump A, Hardisty RE, Blanchard S, Coimbra RS, Perfettini I, Parkinson N, Mallon AM, Glenister P, Rogers MJ, Paige AJ, Moir L, Clay J, Rosenthal A, Liu XZ, et al. (2003) Defects in whirlin, a PDZ domain molecule involved in stereocilia elongation cause deafness in the whirler mouse and families with DFNB31. Nat Genet 34:421-428. CrossRef Medline

Mentis GZ, Blivis D, Liu W, Drobac E, Crowder ME, Kong L, Alvarez FJ, Sumner CJ, O’Donovan MJ (2011) Early functional impairment of sensory-motor connectivity in a mouse model of spinal muscular atrophy. Neuron 69:453-467. CrossRef Medline

Ottoson D (1964) The effect of sodium deficiency on the response of the isolated muscle spindle. J Physiol 171:109-118. CrossRef Medline

Pierrot-Deseilligny E, Burke D (2005) The circuitry of the human spinal cord. Cambridge: Cambridge UP.

Poole K, Herget R, Lapatsina L, Ngo HD, Lewin GR (2014) Tuning Piezo ion channels to detect molecular-scale movements relevant for fine touch. Nat Commun 5:3520. CrossRef Medline

Prost J, Barbetta C, Joanny JF (2007) Dynamical control of the shape and size of stereocilia and microvilli. Biophys J 93:1124-1133. CrossRef Medline

Safieddine S, El-Amraoui A, Petit C (2012) The auditory hair cell ribbon synapse: from assembly to function. Annu Rev Neurosci 35:509-528. CrossRef Medline

Shneider NA, Mentis GZ, Schustak J, O’Donovan MJ (2009) Functionally reduced sensorimotor connections form with normal specificity despite abnormal muscle spindle development: the role of spindle-derived neurotrophin 3. J Neurosci 29:4719-4735. CrossRef Medline

Simon A, Shenton F, Hunter I, Banks RW, Bewick GS (2010) Amiloridesensitive channels are a major contributor to mechanotransduction in mammalian muscle spindles. J Physiol 588:171-185. CrossRef Medline

Takahashi K, Ishida M, Takahashi H (2009) Expression of Sema3D in subsets of neurons in the developing dorsal root ganglia of the rat. Neurosci Lett 455:17-21. CrossRef Medline

Talbot WH, Darian-Smith I, Kornhuber HH, Mountcastle VB (1968) The sense of flutter-vibration: comparison of the human capacity with response patterns of mechanoreceptive afferents from the monkey hand. J Neurophys 31:301-334.

van Wijk E, van der Zwaag B, Peters T, Zimmermann U, Te Brinke H, Kersten FF, Märker T, Aller E, Hoefsloot LH, Cremers CW, Cremers FP, Wolfrum U, Knipper M, Roepman R, Kremer H (2006) The DFNB31 gene product whirlin connects to the Usher protein network in the cochlea and retina by direct association with USH2A and VLGR1. Hum Mol Genet 15:751-765. CrossRef Medline

Wellnitz SA, Lesniak DR, Gerling GJ, Lumpkin EA (2010) The regularity of sustained firing reveals two populations of slowly adapting touch receptors in mouse hairy skin. J Neurophysiol 103:3378-3388. CrossRef Medline

Windhorst U (2007) Muscle proprioceptive feedback and spinal networks. Brain Res Bull 73:155-202. CrossRef Medline

Woo SH, Ranade S, Weyer AD, Dubin AE, Baba Y, Qiu Z, Petrus M, Miyamoto T, Reddy K, Lumpkin EA, Stucky CL, Patapoutian A (2014) Piezo2 is required for Merkel-cell mechanotransduction. Nature 509: 622-626. CrossRef Medline

Xiong W, Grillet N, Elledge HM, Wagner TF, Zhao B, Johnson KR, Kazmierczak P, Müller U (2012) TMHS is an integral component of the mechanotransduction machinery of cochlear hair cells. Cell 151:1283-1295. CrossRef Medline

Yang J, Liu X, Zhao Y, Adamian M, Pawlyk B, Sun X, McMillan DR, Liberman MC, Li T (2010) Ablation of Whirlin long isoform disrupts the USH2 protein complex and causes vision and hearing loss. PLoS Genet 6:e1000955. CrossRef Medline 